\title{
Spatially resolved hard X-ray polarization in solar flares: effects of Compton scattering and bremsstrahlung
}

\author{
N. L. S. Jeffrey and E. P. Kontar
}

School of Physics \& Astronomy, University of Glasgow, G12 8QQ, UK

Received 31 August 2011 / Accepted 28 October 2011

\begin{abstract}
Aims. We study the polarization of hard X-ray (HXR) sources in the solar atmosphere, including Compton backscattering of photons in the photosphere (the albedo effect) and the spatial distribution of polarization across the source.

Methods. HXR photon polarization and spectra produced via electron-ion bremsstrahlung emission are calculated from various electron distributions typical for solar flares. Compton scattering and photoelectric absorption are then modelled using Monte Carlo simulations of photon transport in the photosphere to study the observed (primary and albedo) sources. Polarization maps across HXR sources (primary and albedo components) for each of the modelled electron distributions are calculated at various source locations from the solar centre to the limb.

Results. We show that Compton scattering produces a distinct polarization variation across the albedo patch at peak albedo energies of 20-50 keV for all anisotropies modelled. The results show that there are distinct spatial polarization changes in both the radial and perpendicular to radial directions across the extent of the HXR source at a given disk location. In the radial direction, the polarization magnitude and direction at specific positions along the HXR source will either increase or decrease with increased photon distribution directivity towards the photosphere. We also show how high electron cutoff energies influence the direction of polarization at above $\sim 100 \mathrm{keV}$.

Conclusions. Spatially resolved HXR polarization measurements can provide important information about the directivity and energetics of the electron distribution. Our results indicate the preferred angular resolution of polarization measurements required to distinguish between the scattered and primary components. We also show how spatially resolved polarization measurements could be used to probe the emission pattern of an HXR source, using both the magnitude and the direction of the polarization.
\end{abstract}

Key words. Sun: flares - Sun: X-rays, gamma rays - polarization - X-rays: general - Sun: activity - scattering

\section{Introduction}

Solar flare accelerated electrons can be studied via their radiation using various emission mechanisms. Electrons with energies from tens to hundreds of $\mathrm{keV}$ can reach the denser layers of the solar chromosphere and produce strong hard X-ray (HXR) emission via bremsstrahlung; the properties of which tell us about the energetics and directivity of the accelerated electron distribution. A major insight into the angular properties of the photon distribution and hence the radiating electron distribution can come directly from the polarization of the HXRs. Since the anisotropy and polarization of the photon distribution produced by bremsstrahlung will increase with the anisotropy of the electron distribution (e.g. Brown 1972; Haug 1972; Leach \& Petrosian 1983), then in theory HXR polarization is one of the direct tools available to infer the anisotropy of the emitting electron distribution. However, compared with the other HXR observables: energy, spatial location, source size, time of arrival etc., polarization measurements through the years (see Kontar et al. 2011, as a review) have been fraught with difficulties and the measurements obtained have often been met with skepticism. Nonetheless many missions have reported measurements of HXR polarization from solar flares (Tindo et al. 1970, 1972; Nakada et al. 1974; Tindo et al. 1976; Lemen et al. 1982; McConnell et al. 2004; Boggs et al. 2006; Suarez-Garcia et al. 2006; McConnell et al. 2007).

Another valuable tool for determining the directivity of the photon distribution is the Compton backscattered (albedo) component. Photons emitted towards the photosphere can Compton scatter back out towards the observer and be detected alongside the primary photons directly emitted from the source (Tomblin 1972; Santangelo et al. 1973). Even for an isotropic source (minimum albedo), the albedo component can account for as much as $40 \%$ of the observed flux at peak albedo energies of 20-50 keV (Bai \& Ramaty 1978; Kontar et al. 2006) and for high downward beaming, albedo can dominate the observed flux at certain energies. Separating the albedo component from the directly emitted primary component is essential as it provides us with a unique (and only for individual flares) opportunity of inferring the properties of the photon distribution directed towards the photosphere (Kontar \& Brown 2006; Battaglia \& Kontar 2011), which otherwise would be poorly known. The albedo component can also change various measurable properties of HXR sources, from producing a distinctive change in the photon spectrum between $\sim 10-100 \mathrm{keV}$ (Bai \& Ramaty 1978) to increasing source sizes and shifting the position of sources (Kontar \& Jeffrey 2010). Compton scattering is also polarization dependent and hence the polarization of an HXR source will be altered by the albedo component (Henoux 1975; Langer \& Petrosian 1977; Bai \& Ramaty 1978). The albedo component will not only change the polarization of the entire source but it will also produce spatial variations in polarization across the extent of the albedo source.

Both HXR polarization and albedo measurements contribute information regarding the directivity of the photon distribution and hence allow us to deduce the pitch angle distribution of the 
X-ray emitting electrons (e.g. Kontar et al. 2011, as a review). In addition, the direction of the polarization vector is related to the direction of electron beaming and hence the dominant direction of electrons in a loop (Emslie et al. 2008). Spatially resolved polarization measurements across an HXR source caused by albedo, have the advantage over spatially integrated measurements because both the magnitude and the direction of polarization will change with photon directivity and allow us to map the albedo and primary components. Therefore understanding how these two parameters change with the photon anisotropy is essential and provides us with a new method of investigating the entire photon anisotropy from a single HXR source.

Upcoming missions such as the Gamma-Ray Polarimeter/ Imager for Solar flares (GRIPS) (Shih et al. 2009) (which is planned to be flown on a balloon in 2012) or other astrophysical missions (Bloser et al. 2009; Muleri et al. 2009) may be able to provide us with spatially resolved polarization measurements. Though GRIPS will have an angular resolution of $\sim 12^{\prime \prime}$ available at energies as low as $\sim 25 \mathrm{keV}$, it is doubtful that the GRIPS polarimeter will be able to measure polarization at peak albedo energies of 20-50 keV, due to photoelectric absorption dominating over Compton scattering in the detector at these energies, but hopefully some technologies involved in GRIPS can be used for future missions. Nevertheless, although there are a number of simulations for the spatially integrated polarization signal in flares (Elwert \& Haug 1970; Haug 1972; Langer \& Petrosian 1977; Bai \& Ramaty 1978; Zharkova et al. 1995; Emslie et al. 2008; Zharkova et al. 2010), there are currently no theoretical predictions or modelled spatially resolved HXR polarization maps. In addition, the effect of X-ray albedo on polarization signatures is often ignored in the models.

In this paper, we compute spatially resolved polarization across HXR sources at various locations on the solar disk, taking into account the influence of albedo, for various emitting electron populations. We also examine how changing the maximum electron energy available during bremsstrahlung can alter polarization measurements with photon energy, possibly providing a diagnostic for the maximum electron cutoff energy.

\section{Bremsstrahlung interactions and the creation of the photon distribution}

\subsection{Stokes parameters and defining photon polarization}

The polarization state of incoherent radiation can be completely described using four Stokes parameters (Stokes 1852; Chandrasekhar 1960). The Stokes vector consists of these four parameters and takes the form of $S=[I, Q, U, V]$. The first Stokes parameter $I$ is the normalised total intensity of the photon beam, while $Q / I, U / I$ and $V / I$ will have values between -1 and 1 . The second and third normalised Stokes parameters are used to define linear polarization with 1 or -1 indicating that the beam/photon packet is completely polarized with the sign providing the direction of polarization. The fourth parameter is used to describe circular polarization but bremsstrahlung emission in the solar corona, or Compton scattering only produces radiation that is linearly polarized. In order to produce circularly polarised radiation via bremsstrahlung, the spins of the radiating electrons need to be aligned and the magnetic field in the corona/chromosphere is obviously not strong enough for this alignment. This means that only the first three Stokes parameters are required and the fourth can be set to zero throughout our simulations. Generally in X-ray and gamma ray astronomy the polarization of radiation is measured using the degree of polarization (DOP) and the polarization angle $\Psi$, which is the preferred direction of the electric field. These are defined using the Stokes parameters as,

$\mathrm{DOP}=\frac{\sqrt{Q^{2}+U^{2}}}{I}$

and

$\Psi=\frac{1}{2} \arctan \left(\frac{-U}{-Q}\right)$,

where the angle $\Psi$ is chosen to lie within the quadrant between $\left[-180^{\circ}, 180^{\circ}\right]$, so that $\arctan \left(\frac{+0}{+0}\right)=+0, \arctan \left(\frac{+0}{-0}\right)=$ $+180^{\circ}, \arctan \left(\frac{-0}{+0}\right)=-0$ and $\arctan \left(\frac{-0}{-0}\right)=-180^{\circ}$. The negatives introduced into Eq. (2) ensure that a negative $Q$ gives 0 and a positive $Q$ gives $90^{\circ}$. Hence with this definition, when $\Psi=0^{\circ}$, the observed radiation is polarized parallel to the radial direction at the solar disk and when $\Psi=90^{\circ}$, the radiation is polarized perpendicular to the radial direction. The opposite definition (i.e. $\Psi=90^{\circ}$ - polarized parallel to the radial direction) would have been equally valid as long as definitions throughout the simulations are consistent.

We notice that the Stokes parameters are also frame dependent and hence have to be updated by the use of rotation matrices (Hovenier \& van der Mee 1983) when moving between different coordinate frames. The rotations used in our simulations when moving between the source frame and the scattering frame and vice versa are shown in Appendices A and B. The DOP remains unchanged by the rotation but the polarization angle $\Psi$ is measured with respect to the new frame.

For simplicity, the flare loop and hence the dominant direction of electrons is always assumed to lie parallel with the local solar vertical, i.e. no tilt (see Emslie et al. 2008, for non-zero tilt). This means that for the bremsstrahlung emission, the polarization direction $\Psi$ across the entire source (source assumed to be small in comparison with the solar disk) and at spatially resolved positions across the source can only ever equal $0^{\circ}$ or $90^{\circ}$, since $U$ from the bremsstrahlung emission is always close to zero (Bai \& Ramaty 1978). Since in the solar disk frame (the observed frame of the HXR source), Compton scattering can produce values of $U$ other than 0 , this means that the Compton scattered $\Psi$ can have values other than $0^{\circ}$ or $90^{\circ}$. This is true for the spatially resolved polarization of the albedo (and hence the total observed) source, while the spatially integrated albedo Stokes parameters again sum to produce $\Psi$ values of either $0^{\circ}$ or $90^{\circ}$, for a nontilted loop.

\subsection{Primary photon distribution}

The intensity of the photon distribution $I(\epsilon, \theta)$ produced by bremsstrahlung for a chosen electron distribution $F(E, \beta)$ is given by

$I(\epsilon, \theta) \propto \int_{E=\epsilon}^{\infty} \int_{\Phi=0}^{2 \pi} \int_{\beta=0}^{\pi} F(E, \beta) \sigma_{I}(E, \epsilon, \Theta) \sin \beta \mathrm{d} \beta \mathrm{d} \Phi \mathrm{d} E$

where $\epsilon$ is the photon energy, $E$ is the electron energy, $F(E, \beta)$ is the target electron flux density differential in energy and $\sigma_{I}(E, \epsilon, \Theta)$ is the total (averaged over all polarization states) angle-dependent bremsstrahlung cross-section (Elwert \& Haug 1970; Bai \& Ramaty 1978; Massone et al. 2004). $\theta \in\left[0^{\circ}, 180^{\circ}\right]$ is the photon polar emission angle measured from the local solar vertical with $\theta=0^{\circ}$ directed away from the Sun. $\beta \in\left[0^{\circ}, 180^{\circ}\right]$ 
is the pitch angle of the emitting electrons velocity to the local magnetic field with $\beta=0^{\circ}$ also directed away from the Sun. $\Phi \in\left[0^{\circ}, 360^{\circ}\right]$ is the corresponding electron azimuthal angle and $\Theta(\beta, \Phi, \theta)$ is the angle between the plane of emission (at angle $\beta$ ) and the plane of observation (at angle $\theta$ ). The photon emission is described by $\mu=\cos \theta$, where $\mu$ from 0 to 1 corresponds to emission away from the Sun, and $\mu$ from -0 to -1 corresponds to emission towards the solar surface. The photon emission angle $\mu=\cos \theta$ is related to the electron pitch angle $\beta$ by:

$\cos \Theta=\cos \theta \cos \beta+\sin \theta \sin \beta \cos \Phi$.

Viewing the outward emission from $\mu=0.0-1.0$ corresponds to observing the source at a selected heliocentric angle on the solar disk, i.e. $\mu=0.0$ would correspond to $90^{\circ}$ and is equivalent to viewing a source sitting at the solar limb. The other Stokes parameters $Q$ and $U$ can be calculated in a similar manner:

$$
Q(\epsilon, \theta) \propto \int_{E=\epsilon}^{\infty} \int_{\Phi=0}^{2 \pi} \int_{\beta=0}^{\pi} F(E, \beta) \sigma_{Q}(E, \epsilon, \Theta) \sin \beta \mathrm{d} \beta \mathrm{d} \Phi \mathrm{d} E,
$$

$$
U(\epsilon, \theta) \propto \int_{E=\epsilon}^{\infty} \int_{\Phi=0}^{2 \pi} \int_{\beta=0}^{\pi} F(E, \beta) \sigma_{U}(E, \epsilon, \Theta) \sin \beta \mathrm{d} \beta \mathrm{d} \Phi \mathrm{d} E
$$

with the only difference being the use of either $\sigma_{I}(E, \epsilon, \Theta)$, $\sigma_{Q}(E, \epsilon, \Theta)$ or $\sigma_{U}(E, \epsilon, \Theta)$. $Q(\epsilon, \theta)$ and $U(\epsilon, \theta)$ are then normalised between $[-1,1]$ by dividing through by $I(\epsilon, \theta)$. $\sigma_{I}(E, \epsilon, \Theta), \sigma_{Q}(E, \epsilon, \Theta)$ and $\sigma_{U}(E, \epsilon, \Theta)$ are the polarization dependent cross-sections for bremsstrahlung taken from Gluckstern \& Hull (1953) and also following the form used in Haug (1972) and Emslie et al. (2008), are given by

$\sigma_{I}(E, \epsilon, \Theta)=\sigma_{\perp}(E, \epsilon, \Theta)+\sigma_{\|}(E, \epsilon, \Theta)$,

$\sigma_{Q}(E, \epsilon, \Theta)=\left(\sigma_{\perp}(E, \epsilon, \Theta)-\sigma_{\|}(E, \epsilon, \Theta)\right) \cos 2 \Theta$

and

$\sigma_{U}(E, \epsilon, \Theta)=\left(\sigma_{\perp}(E, \epsilon, \Theta)-\sigma_{\|}(E, \epsilon, \Theta)\right) \sin 2 \Theta$,

where $\sigma_{\perp}(E, \epsilon, \Theta)$ and $\sigma_{\|}(E, \epsilon, \Theta)$ are the perpendicular and parallel components of the bremsstrahlung cross-section respectively.

\subsubsection{Electron distribution}

In our simulations the X-ray emitting electron distribution is chosen to have the form,

$$
F(E, \beta) \propto E^{-\delta} \exp \left(-\frac{(1+\cos \beta)^{2}}{\Delta v^{2}}\right) .
$$

The energy dependence follows a power law as shown by observations (see Holman et al. 2011; Kontar et al. 2011, as recent reviews) and the electron angular distribution is a Gaussian. The use of a Gaussian allows the angular anisotropy of the electron distribution to be easily controlled by a single parameter $\Delta v$. The smaller the value of $\Delta v$, the greater the proportion of the electron distribution, and hence the resulting photon emission directed towards the photosphere.

In all simulations shown in this paper, we used a typical solar flare with $\delta=2$ (spectral index of the mean electron spectrum) and $\Delta v=4.0, \Delta v=0.5$ or $\Delta v=0.1$ to describe various pitch angle distributions of electrons. Note that a target electron distribution of $\delta=2$ is produced by an injected electron distribution of $\delta_{\text {inj }}=\delta+2=4$ (see Holman et al. 2011, as a review). A $\Delta v=4.0$ electron distribution produces an approximately isotropic, unpolarised photon distribution, while the $\Delta v=0.5$ and $\Delta v=0.1$ electron distributions produce photon distributions with greater and greater beaming towards the photosphere. The resulting fluxes and polarizations of each component [primary towards the observer only (orange), albedo (blue) and the total observed emission (green)] plotted against emission/heliocentric angle $\mu=\cos \theta$ are shown for each of the $\Delta v=4.0, \Delta v=0.5$ and $\Delta v=0.1$ photon distributions in Fig. 1. The resulting photon distributions in Fig. 1 are shown for the energies of 20$50 \mathrm{keV}$, where the albedo emission peaks. As expected, even though the $\Delta v=0.1$ distribution has a greater downward beaming (and hence a smaller observer directed emission) than the $\Delta v=0.5$ distribution, within this low energy band the difference in anisotropy between the two primary distributions cannot be clearly seen, however differences between the two distributions can be clearly observed in the DOP with disk location over this energy range (second row Fig. 1).

An HXR source is modelled at a chosen height $h$ in the chromosphere. For the HXR energy range, the source is about $1 \mathrm{Mm}$ above the photosphere in accordance with recent X-ray observations (Kontar et al. 2008; Prato et al. 2009; Saint-Hilaire et al. 2010; Mrozek \& Kowalczuk 2010; Kontar et al. 2010; Battaglia \& Kontar 2011). The source spatial extent is modelled using a two-dimensional circular Gaussian distribution,

$I(x, y) \propto \exp \left(-\frac{x^{2}}{2 d^{2}}-\frac{y^{2}}{2 d^{2}}\right)$

where $d$ is the size of the HXR source. Source sizes will be defined by the (Gaussian) Full Width Half Maximum ( $F W H M)$, given by $F W H M \equiv \sqrt{8 \ln (2)} d$.

\section{Monte Carlo simulations of photon transport}

A Monte Carlo code developed by Kontar \& Jeffrey (2010) was modified to include polarization (see Appendices A and B, for details). The energy, angular and polarization properties of the input photon distributions for the MC code are determined via the chosen input electron distributions given by Eq. (10), while the spatial properties are determined via Eq. (11). To input $I(\epsilon, \theta), Q(\epsilon, \theta)$ and $U(\epsilon, \theta)$ (Eqs. (3), (5) and (6)) into our MC simulations, distributions of energy $\epsilon$, angle $\theta$ and polarization $Q$ and $U$ are numerically created from $I(\epsilon, \theta), Q(\epsilon, \theta)$ and $U(\epsilon, \theta)$. Each photon azimuthal angle $\phi$ is simply drawn from a uniform, random distribution between 0 and $2 \pi$. To achieve the best statistics possible, we use $10^{8}$ photons in every MC code run. Since the photon angular distribution is measured from the local solar vertical, photons with $\theta<90^{\circ}$ escape from the solar atmosphere as the primary emission and photons with $\theta \geq 90^{\circ}$ will travel towards the photosphere, creating the albedo emission. Photons are allowed to move freely until they reach the photosphere, where they interact with free or bound electrons. In the simulations the photosphere is just defined by having a Hydrogen number density of $n_{\mathrm{H}}=1.16 \times 10^{17} \mathrm{~cm}^{-3}$ (Vernazza et al. 1981). Here the photons can no longer travel without interacting in some way with the photospheric material. Each photon moves a step-size before an interaction and this is calculated using $s s=-l \ln \zeta_{\text {step }}$, where $l$ is the photon mean free path and $\zeta_{\text {step }} \in[0,1]$. The photon mean free path is calculated by $l=1 / n_{\mathrm{H}} \sigma_{\text {total }}$, where $\sigma_{\text {total }}=\sigma_{\mathrm{c}}+\sigma_{\mathrm{a}}$, the addition 

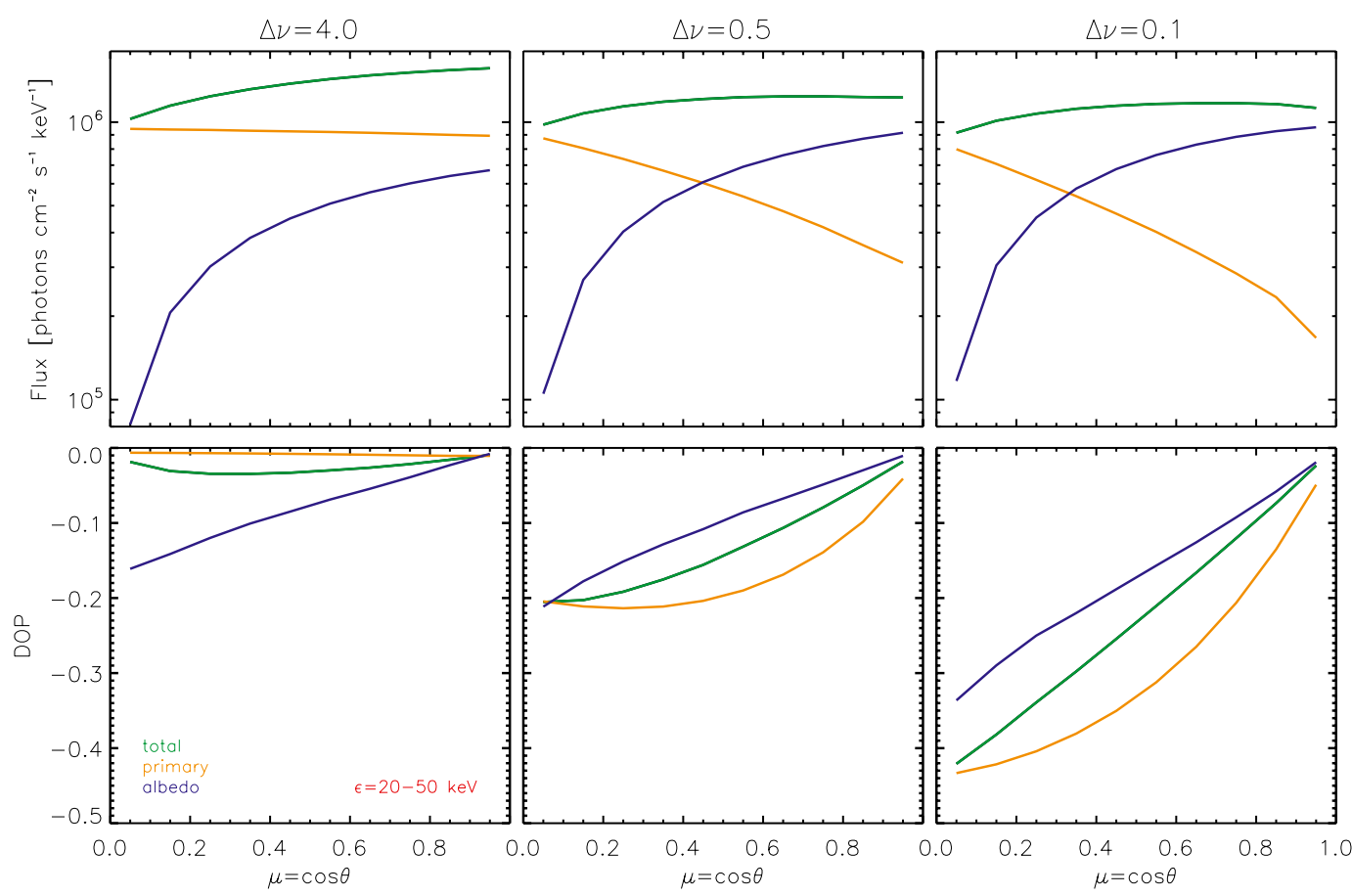

Fig. 1. Photon flux (top row) and DOP (bottom row) for upward primary (orange), albedo (blue) and total (green) components across the entire source for the $\Delta v=4.0$ (1st column), the $\Delta v=0.5$ (2nd column) and the $\Delta v=0.1$ (last column) created photon distributions respectively, at the peak albedo energies of $20-50 \mathrm{keV}$, for different locations on the solar disk from the centre $(\mu=1.0)$ to the limb $(\mu=0.0)$. Note here that a negative DOP denotes that the direction of polarization $\Psi$ across the source is parallel to the radial direction. The DOP for $\Delta v=4.0$ photon distribution (near isotropic distribution) shows the same result as Bai \& Ramaty (1978), as expected.

of the Compton scattering cross-section (using the form shown in Bai \& Ramaty 1978) and photoelectric absorption cross section. This photon mean free path, $l$, is of the order $100 \mathrm{~km}$. A photon interaction with the photospheric medium can either be by Compton scattering or by photoelectric absorption. For photons with energies less than $\sim 10 \mathrm{keV}$, photoelectric absorption is the more probable process while scattering dominates above $\sim 10 \mathrm{keV}$ (Fig. 2). When a photon is absorbed, it is removed from the simulations. For each photon, one of the two processes is chosen by calculating the ratio of $\sigma_{\mathrm{c}} / \sigma_{\text {total }}$. Another random number $\zeta_{\text {pick }}$ is then sampled from a uniform distribution between 0 and 1 . If the ratio is greater than $\zeta_{\text {pick }}$ then the photon is Compton scattered and if the ratio is less than $\zeta_{\text {pick }}$, then the photon is absorbed. The curvature of the Sun is included in these simulations and a photon exits the photosphere when it satisfies the condition $z>z_{\odot}=\sqrt{R_{\odot}^{2}-x^{2}-y^{2}}-R_{\odot}$, where $R_{\odot}$ is the radius of the Sun (taken to be $6.96 \times 10^{10} \mathrm{~cm} \sim 960^{\prime \prime}$ ). The extent of the albedo patch is limited by properly modelling the curvature of the Sun. The photons are allowed to scatter multiple times until they exit the photosphere or are removed via absorption. Most photons will leave the photosphere during the first scatter, with subsequent scatterings producing less and less photons (this is shown in terms of Green's functions in Kontar et al. 2006). Photons that exit the photosphere with $\cos \theta>0$ can then be collected into selected angular or energy bins corresponding to HXR sources sitting at any chosen heliocentric angle on/above the solar disk. In these simulations, source positions and sizes of each component (primary and albedo) and the total source are given by the first and second moments of each intensity distribution (Kontar \& Jeffrey 2010), which are the mean $(\bar{x}, \bar{y})$ and variance $\left(\operatorname{var}_{x}, \operatorname{var}_{y}\right)$ respectively. Though the primary distribution is initially Gaussian, the albedo (and hence total observed)

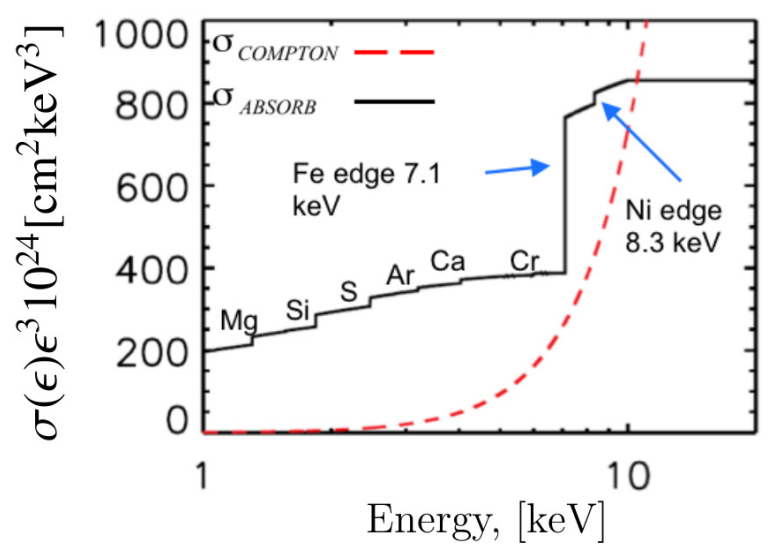

Fig. 2. Absorption $\sigma_{\mathrm{a}}$ (black solid) and Compton $\sigma_{\mathrm{c}}$ (red dashed) crosssections multiplied by $10^{24} \epsilon^{3}(\epsilon$ in $\mathrm{keV})$ at low energies below $10 \mathrm{keV}$. The absorption cross-section is calculated using photospheric element abundances by Asplund et al. (2009).

distribution will have a complex shape that is no longer Gaussian. To quantify the sizes we use "Gaussian" Full Width Half Maximum ( $F W H M$ ) defined as 2.35 times the square root of the second-moment of the spatial distribution (not the actual FWHM of the complex distribution) (see Kontar \& Jeffrey 2010, for details). This allows a simple comparison with measurable parameters. All FWHM values in this paper are measured in this way.

\subsection{Compton scattering}

Above $\sim 10 \mathrm{keV}$, Compton scattering is the dominant process in the photosphere. The polarization dependent differential 
Compton scattering cross-section is given by Klein \& Nishina (1929), but the form used in these simulations is from McMaster (1961) and Bai \& Ramaty (1978)

$$
\frac{\mathrm{d} \sigma_{\mathrm{c}}}{\mathrm{d} \Omega}=\frac{1}{2} r_{0}^{2}\left(\frac{\epsilon}{\epsilon_{0}}\right)^{2}\left(\frac{\epsilon}{\epsilon_{0}}+\frac{\epsilon_{0}}{\epsilon}-\sin ^{2} \theta_{\mathrm{S}}\left(1-Q \cos 2 \phi_{\mathrm{S}}-U \sin 2 \phi_{\mathrm{S}}\right)\right),
$$

where $r_{0}=2.82 \times 10^{-13} \mathrm{~cm}$ is the classical electron radius, $\epsilon_{0}$ is the energy of the incoming photon, $\epsilon$ is the energy of the outgoing photon, $\theta_{\mathrm{S}}$ is the polar scattering angle, $\phi_{\mathrm{S}}$ is the azimuthal scattering angle and $Q$ and $U$ are the linear Stokes parameters respectively (McMaster 1961; Bai \& Ramaty 1978). The maximum change in DOP occurs when $\theta_{\mathrm{S}}=90^{\circ}$ and no change in DOP occurs for a backscattering at $180^{\circ}$. The azimuthal scattering angle $\phi_{\mathrm{S}}$ also has a non uniform dependency on the incoming polarization state (see Appendix B). In the simulations, the Klein-Nishina cross-section is multiplied by $Z_{\text {photo }}=1.18$ to take account of elements higher than Hydrogen that are present within the photosphere. $Z_{\text {photo }}$ indicates the average atomic number and the number of electrons per Hydrogen atom in the photosphere, and is given by

$Z_{\text {photo }}=\frac{\sum_{Z} Z 10^{A_{Z}}}{10^{A_{\mathrm{H}}}}$

where $A_{Z}$ is the $\log _{10}$ abundance of an element with atomic number $Z$ relative to Hydrogen while $A_{\mathrm{H}}=12$ is the $\log _{10}$ abundance of Hydrogen (Asplund et al. 2009). More details regarding the updating of photon parameters $\left(\theta_{\mathrm{S}}, \epsilon, \phi_{\mathrm{S}}, Q, U\right)$ after Compton scattering are given in the appendices (A and $\mathrm{B})$.

\subsection{Photoelectric absorption of $X$-ray photons}

For photons with energies below $\sim 10 \mathrm{keV}$, photoelectric absorption is the most probable photon interaction in the photosphere. The process of absorption is heavily dependent upon the composition of elements and the abundance of these elements within the photosphere. Absorption was therefore modelled using the latest known solar photospheric abundances taken from Asplund et al. (2009). Absorption cross-section codes for the most important elements of $\mathrm{H}, \mathrm{He}, \mathrm{C}, \mathrm{N}, \mathrm{O}, \mathrm{Ne}, \mathrm{Na}, \mathrm{Mg}, \mathrm{Al}, \mathrm{Si}, \mathrm{S}, \mathrm{Cl}$, $\mathrm{Ar}, \mathrm{Ca}, \mathrm{Cr}, \mathrm{Fe}$ and $\mathrm{Ni}$ were adapted from Balucinska-Church \& McCammon (1992). Absorption is much less probable above $10 \mathrm{keV}$ and for energies higher than $10 \mathrm{keV}$, the absorption crosssection was approximated by $\sigma_{\mathrm{a}}\left(\epsilon_{0}\right) \propto \epsilon_{0}^{-3}$. The probability of photoelectric absorption is assumed to be independent of polarization (Poutanen et al. 1996). Only the angular distribution of the ejected electron is dependent upon the photon polarization, which is not modelled in our simulations. A comparison of the Compton scattering and absorption cross sections is shown in Fig. 2 (with $\sigma_{\mathrm{a}}$ and $\sigma_{\mathrm{c}}$ multiplied by $10^{24} \epsilon^{3}(\epsilon$ in $\mathrm{keV}$ ) for comparison with Morrison \& McCammon 1983). Any differences between Fig. 2 and Morrison \& McCammon (1983) are due to the newer element abundances (Asplund et al. 2009) and updated absorption cross-section codes (in particular helium) being used in our simulations.

\section{Spatial distribution of X-ray polarization}

\subsection{Single Compton scatter for an isotropic unpolarised source}

In order to demonstrate the spatial variation in polarization due to Compton scattering, the easiest example to consider is the

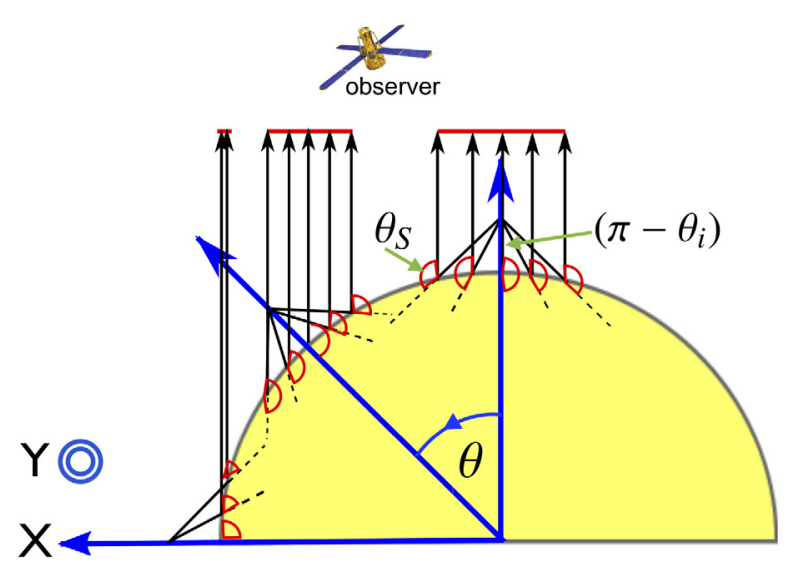

Fig. 3. Diagram of a single Compton scattering in the photosphere for three heliocentric angles of $0^{\circ}, 45^{\circ}$ and $90^{\circ}$. For a single scatter, the DOP tends to $100 \%$ as the scattering angle approaches $90^{\circ}$, producing a variation in polarization across the extent of the source.

albedo patch created by an initially isotropic, unpolarised point source at a height $h$ above the photosphere (see Kontar \& Jeffrey 2010). For this example, the variation in polarization across the source can be described analytically by (McMaster 1961) and assuming no energy losses,

$\mathrm{DOP}=\frac{1-\cos ^{2} \theta_{\mathrm{S}}}{1+\cos ^{2} \theta_{\mathrm{S}}}$

where

$\cos \theta_{\mathrm{S}}=\cos \theta \cos \left(\pi-\theta_{i}\right)+\sin \theta \sin \left(\pi-\theta_{i}\right) \cos \phi$.

$\theta_{\mathrm{S}}$ is the scattering angle, $\theta_{i}$ is the emission angle measured from the local solar vertical, $\theta$ is the heliocentric angle on the solar disk and $\phi$ is the angle measured in the solar disk plane. In Eq. (14), the scattering angle $\theta_{\mathrm{S}}$ determines the DOP. $\theta_{\mathrm{S}}$ is related to distance $r$ from the centre of the albedo patch by Eq. (15) though $r=h \tan \left(\pi-\theta_{i}\right)$, and hence the DOP at any point across the albedo patch sitting at any heliocentric angle on the solar disk can be easily calculated. Note that for this simple example, Eq. (14) assumes that the energy difference between the incoming and scattered photon is negligible, though this is only the case for low HXR energies of $\sim 10 \mathrm{keV}$. The DOP for all photon energies is calculated using the $T$ scattering matrix, which is used in the simulations (Eq. (B.3) in Appendix B) (McMaster 1961).

Figure 3 shows a cartoon of this single scattering from an isotropic point source for three different heliocentric angles of $0^{\circ}, 45^{\circ}$ and $90^{\circ}$. For a source located exactly above the solar centre $\left(0^{\circ}\right)$ at a height $h$, the resulting variation across the photospheric albedo patch is radially symmetric. As the radial distance $r$ from the source centre increases, the scattering angle $\theta_{\mathrm{S}}$ of any observed radiation will decrease from $180^{\circ}$ towards $90^{\circ}$, causing the DOP to grow from $0 \%$ to $\sim 100 \%$. Radiation scattered in the photosphere at a location directly below the source $\left(180^{\circ}\right.$ backscatter) and emitted towards the observer will experience no change in its DOP. This statement is true for sources at any heliocentric angle $\theta_{i}$ but projection effects at angles $\theta_{i}>0^{\circ}$ will create an asymmetry in the polarization pattern along the radial direction, whereas the polarization pattern in the perpendicular to radial direction always remains symmetrical. The described pattern can be seen in Fig. 4 which shows the 

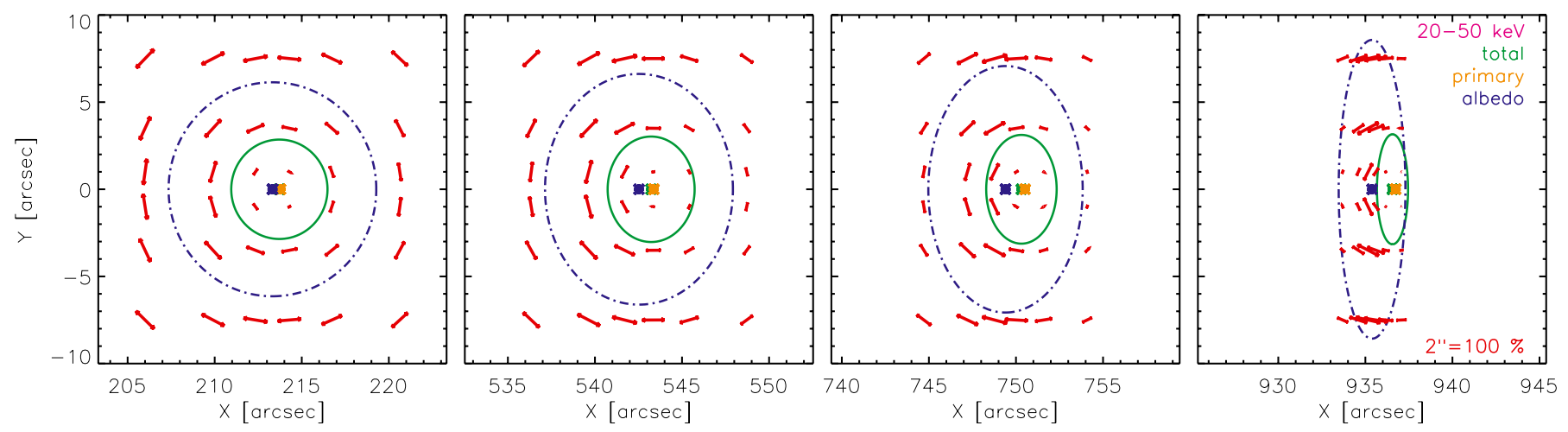

Fig. 4. Albedo polarization maps for an isotropic, unpolarised point source sitting above the photosphere at four different radial locations of $X=214^{\prime \prime}, 543^{\prime \prime}, 750^{\prime \prime}, 936^{\prime \prime}$ at $Y=0^{\prime \prime}$ (corresponding $\mu=0.97,0.82,0.62,0.22$ ) after a single Compton scatter in the photosphere. All results are shown at the peak albedo energies of 20-50 keV. The length of each red arrow indicates the DOP and the direction of each arrow depicts the polarization angle $\Psi$ within the chosen plotting bin. The solar radial direction (or $X$ axis for this case) is defined as the $\Psi=0^{\circ}$ position. An arrow length of 2 " corresponds to a maximum DOP of $100 \%$. The green and blue ellipses give the $F W H M$ of the total and albedo sources respectively, while the green, blue and orange asterisks give the centroid position of the total, albedo and primary sources.
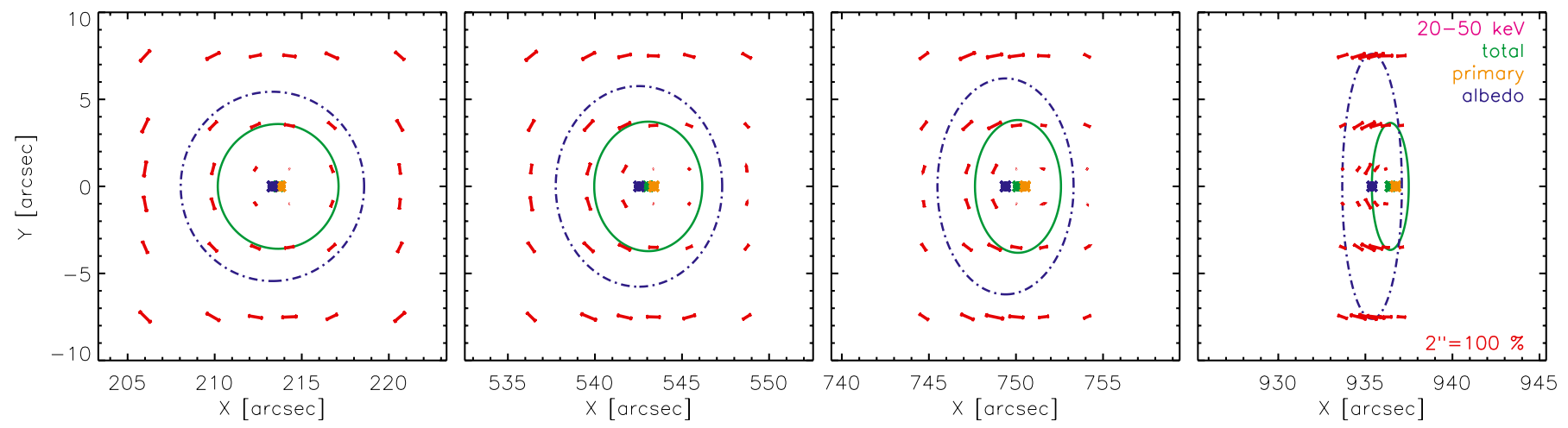

Fig. 5. Albedo polarization maps as in Fig. 4, but for the case of multiple Compton scatterings in the photosphere. Multiple scatterings have acted to decrease the DOP at all points across each source.

polarization maps for a single Compton scattering at four disk locations ranging from the solar centre to the limb, along $Y=0^{\prime \prime}$ at $X=214^{\prime \prime}, 543^{\prime \prime}, 750^{\prime \prime}, 936^{\prime \prime}$. These locations are equivalent to $\mu=0.97,0.82,0.62,0.22\left(\theta_{i}=14^{\circ}, 35^{\circ}, 52^{\circ}, 77^{\circ}\right)$ and denote the approximate positions of the total source, which are shifted from the primary position due to the albedo component (Kontar \& Jeffrey 2010). The polarization across the source at any location on the solar disk can always be measured with respect to the radial connecting the solar disk centre and the centre of the source. Therefore due to the symmetry of the problem, source locations at $Y=0^{\prime \prime}$ considered in this paper can straightforwardly be applied to any solar disk location. In Fig. 4, the dotted blue ellipse denotes the $F W H M$ of the albedo component, the solid green ellipse denotes the FWHM of the total observed source and the orange, blue and green asterisks indicate the centroid positions of primary, albedo and total observed sources respectively. The polarization angle $\Psi$ follows a steady pattern across all sources. $\Psi$ is always at an angle tangential to the line connecting the desired position and the location of the source centroid position. Hence at disk centre locations the $\Psi$ pattern is also symmetrical across the source.

Figure 5 shows the same albedo polarization maps as in Fig. 4 but for multiple Compton scatterings. The overall pattern for the DOP and $\Psi$ are preserved but due to multiple scatterings the overall DOP across all locations over the albedo patch has decreased. A single scattering DOP of $\sim 100 \%$ near the edge of the source has been reduced to $\sim 50 \%$ by multiple scatterings. All subsequent simulations in this paper use multiple Compton scatterings.

The albedo pattern from a primary source at a greater height than $1 \mathrm{Mm}$ (say from a coronal source) should produce the same albedo polarization pattern but over a much greater area in the photosphere. Plotting the polarization pattern at a higher or lower energy than the peak albedo range of $20-50 \mathrm{keV}$ would obscure the albedo polarization pattern due to lowering albedo fluxes at these energies.

\subsection{Anisotropic source at a height of $h=1 \mathrm{Mm}\left(1.4^{\prime \prime}\right)$ and size of $5^{\prime \prime}$}

For a chosen chromospheric HXR source height of $h=1 \mathrm{Mm}$ $\left(1.4^{\prime \prime}\right)$ and a primary source size of $F W H M \sim 5^{\prime \prime}$, simulations were performed for all three photon distributions created by the $\Delta v=4.0, \Delta v=0.5$ and $\Delta v=0.1$ electron distributions. This height was chosen to match chromospheric HXR source height measurements (Kontar et al. 2008; Prato et al. 2009; SaintHilaire et al. 2010; Mrozek \& Kowalczuk 2010; Kontar et al. 2010; Battaglia \& Kontar 2011). All the results shown here are for the energy range of $20-50 \mathrm{keV}$, where albedo emission peaks, producing the largest distortion to the primary component but the best opportunity for the detection of the albedo component. 
N. L. S. Jeffrey and E. P. Kontar: Spatially resolved hard X-ray polarization in solar flares
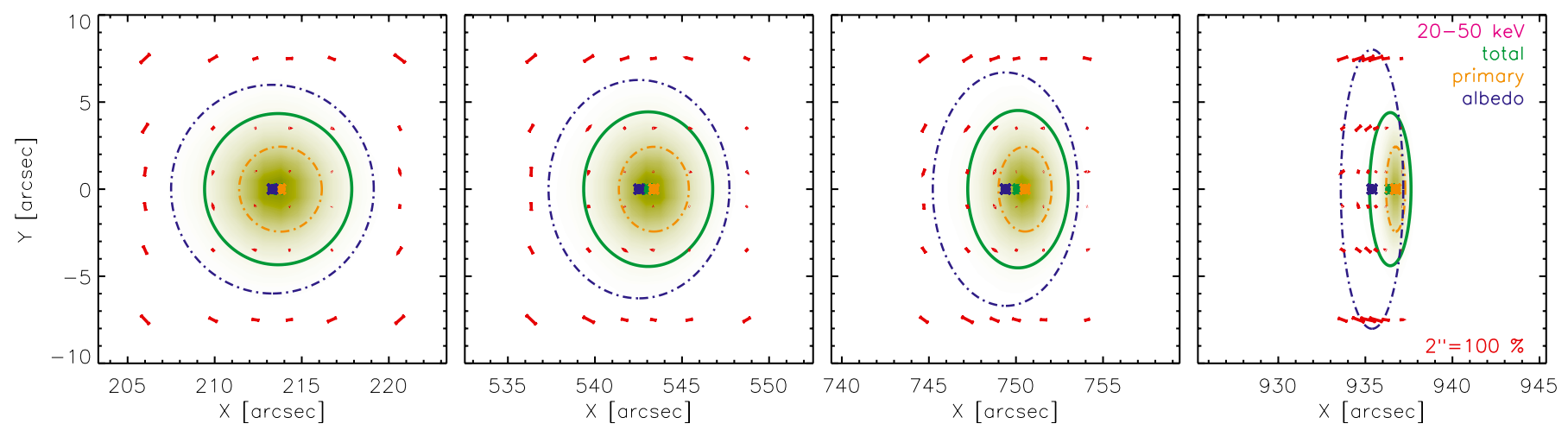

Fig. 6. Total X-ray brightness and polarization (arrows) maps for the photon distribution created by the near isotropic $\Delta v=4.0$ electron distribution for a 5" primary source. The resulting total sources sit at four disk locations of $X=213.6^{\prime \prime}, 542.9^{\prime \prime}, 750.1^{\prime \prime}, 936.6^{\prime \prime}$ at $Y=00^{\prime \prime}$ (corresponding to $\mu \sim 0.97,0.82,0.62,0.22$ ). The green, blue and orange ellipses and asterisks give the $F W H M$ and centroid positions of the total, albedo and primary sources respectively. The primary component is dominant at all disk locations and hence the albedo polarization pattern at small radii (over the extent of the $5^{\prime \prime}$ primary source) is obscured slightly by the flat, constant polarization of the primary source.

Figures 6, 9 and 12 each plot the resulting polarisation maps for four HXR sources (resulting from the primary and albedo components) created by the $\Delta v=4.0, \Delta v=0.5$ and $\Delta v=0.1$ electron distributions respectively. As with Figs. 4 and 5, each figure plots four HXR sources positioned at $\mu \sim$ $0.97,0.82,0.62,0.22$. In Figs. 6, 9 and 12, the dotted ellipses denote the FWHM of the total source (green), the primary source (orange) and the albedo source (blue) and the correspondingly coloured asterisks denote the $(x, y)$ centroid position of the total source and the primary and albedo components respectively. Figures 7, 10 and 13 plot intensity, $I$ (top row), DOP (middle row) and $\Psi$ (bottom row) along the radial direction $X$ centred at $Y=0^{\prime \prime}$ (across a of bin width $=2^{\prime \prime}$ ) for each of the maps in Figs. 6, 9 and 12. Figures 8, 11 and 14 plot the DOP (top row) and $\Psi$ (bottom row) along the perpendicular to radial direction $Y$ centred at $X=213.6^{\prime \prime}, 543.9^{\prime \prime}, 750.1^{\prime \prime}, 936.6^{\prime \prime}$ (again across a bin width $=2^{\prime \prime}$ ) for each of the maps in Figs. 6, 9 and 12.

\subsection{Quasi-isotropic distribution with $\Delta v=4.0$}

The photon distribution produced by the $\Delta v=4.0$ electron distribution is approximately unpolarised and isotropic. Therefore, both spatially integrated and spatially resolved polarization measurements across the primary source at all locations on the solar disk produce DOP $\sim 0 \%$ and $\Psi=0^{\circ}$ (radial) at $20-50 \mathrm{keV}$. The albedo component produces asymmetrical DOP and $\Psi$ variations along the source radial direction (Fig. 7) while along the source perpendicular to radial direction (Fig. 8), variations in albedo DOP and $\Psi$ are approximately symmetrical, since the centroid positions of the primary and albedo components always coincide in the perpendicular to radial direction. The simulated HXR sources plotted in Fig. 6 have a finite source size of $\sim 5^{\prime \prime}$. Compared with a point source (Fig. 5), this produces two main differences: i) photons leave the source from different positions above the photosphere and ii) for certain distributions and disk locations, the primary polarization will dominate over the extent of the primary source. The first property reduces the DOP at all points across the source, compared with the albedo patch created by a point source. Due to the second property, the polarization variation caused by albedo may be slightly masked by the primary component within the source $F W H M$, especially for cases where the primary component is dominant, i.e. isotropic or near isotropic distributions.
For the quasi-isotropic $\Delta v=4.0$ distribution, the primary component is the dominant component at all four disk locations, with the albedo contribution falling as the source location moves towards the limb (Fig. 7 - first row). Hence, the primary component dominates within the $F W H M$ of the total source, while the albedo component dominates after this boundary. The second and third rows of Fig. 7 demonstrate common radial trends in DOP and $\Psi$, not only across the extent of each individual source but also between sources at different disk locations. For a quasi-isotropic distribution, at a particular disk location (other than the disk centre), the highest DOP along the radial direction is observed at the disk-centre-side of the source (where the albedo dominates). This falls to approximately zero within the FWHM of the total source (where the primary dominates) and then increases again towards the limb-side of the source (where the albedo again dominates), but always remains lower than the DOP at the disk-centre-side. Comparing the four disk locations, the DOP at all points along the radial direction of a single source decreases as the source location nears the limb. In the radial direction, spatially resolved DOP can achieve values as high as $\sim 30 \%$ at disk centre locations. The albedo component produces the distinctive $\Psi$ variation shown in Fig. 6. Along the radial direction, $\Psi=90^{\circ}$ at the disk-centre-side of the source, falls to zero within the FWHM extent and then rises again at the limbside of the source. Along the perpendicular to radial direction of a single source (Fig. 8), DOP (first row) and the magnitude of $\Psi$ (second row) are symmetrical due to no projection effects and the centres of the primary and albedo components always coinciding. As with the radial direction, in the perpendicular to radial direction, spatially resolved DOP can achieve values as high as $\sim 30 \%$. While the magnitude of $\Psi$ across the source is symmetrical at each disk location in the perpendicular to radial direction, $\Psi$ itself behaves as an odd function along $Y$, with a $180^{\circ}$ rotational symmetry about the source centre, increasing from the radial at the upper source edge to $|\Psi|>0^{\circ}$ and then back to the radial direction at the lower source edge.

\subsection{Beamed electron distributions $\Delta v=0.5$ and $\Delta v=0.1$}

All plots for the photon distribution created by the $\Delta v=0.5$ electron distribution are shown in Figs. 9-11, while Figs. 12-14 show all plots for the photon distribution created by the $\Delta v=$ 0.1 electron distribution. Comparison of Figs. 6, 9 and 12 

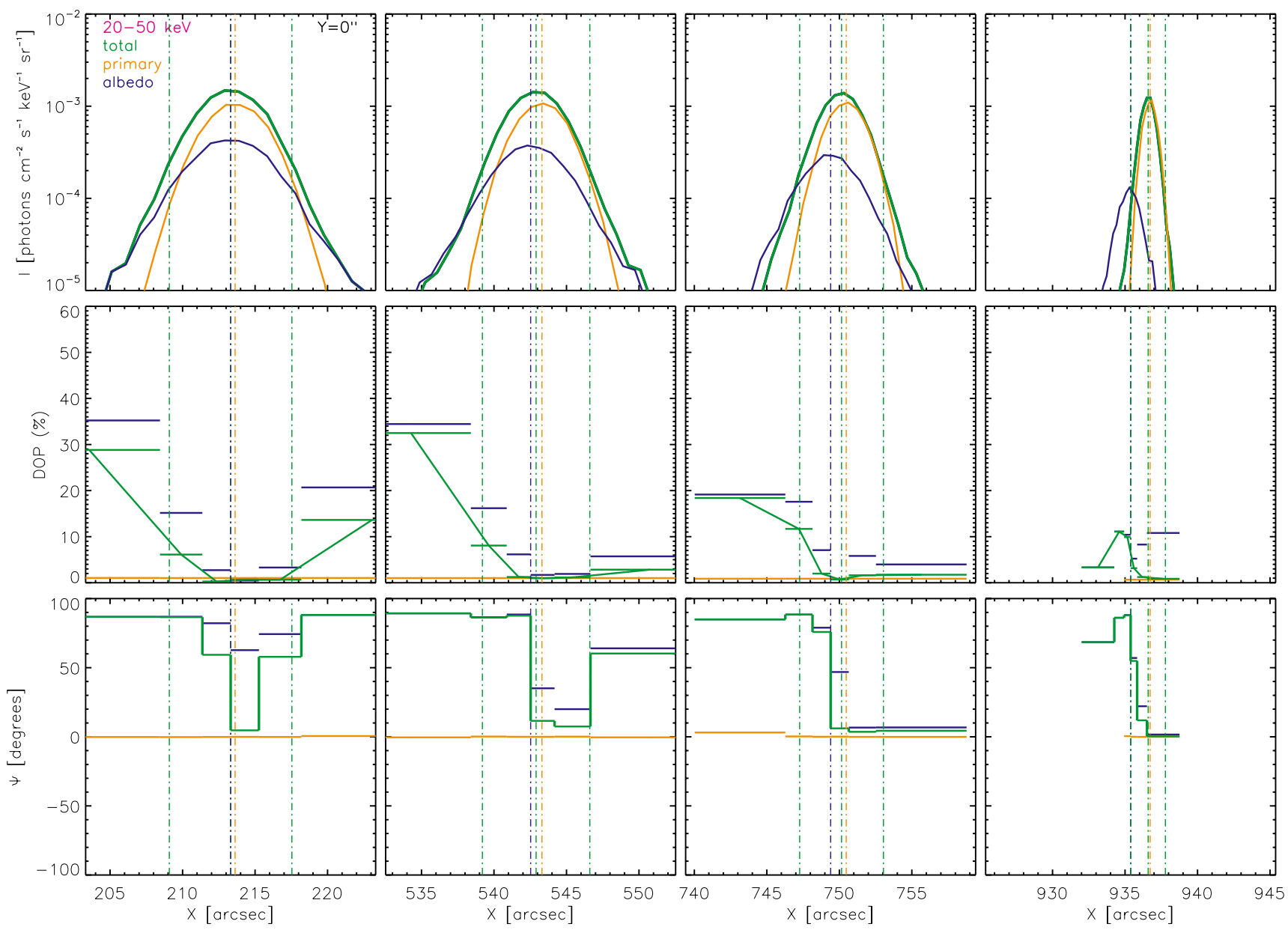

Fig. 7. Radial slices through each of the sources in Fig. 6 for the $\Delta v=4.0$ created photon distribution. Each of the radial slices are taken along $X$ at $Y=0^{\prime \prime}$ for the intensity, $I$, DOP and polarization angle $\Psi$ at the four source locations. As before, orange $=$ primary, blue $=$ albedo and green $=$ total. The green dash-dot lines denote the centroid position and the FWHM of the total observed source while the orange and blue lines denote the centroid positions of the primary and albedo sources respectively. Slices along the radial direction for the $\Delta v=4.0$ distribution show a clear DOP and $\Psi$ pattern at all four disk locations plotted.
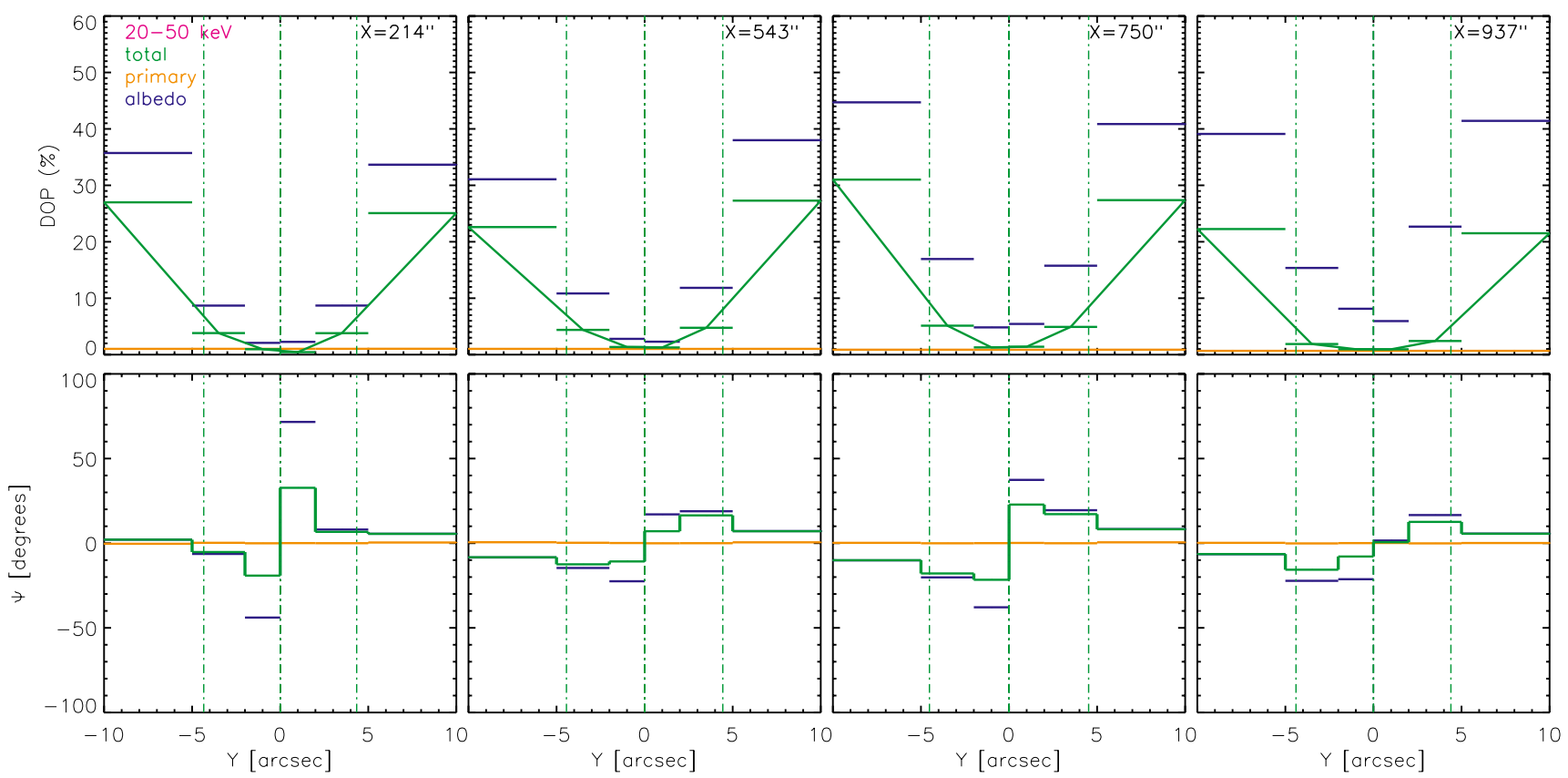

Fig. 8. Perpendicular to radial slices through each of the sources shown in Fig. 6 for the $\Delta v=4.0$ distribution. Each of the perpendicular to radial slices are taken along $Y$ at $X=213.6^{\prime \prime}, 542.9^{\prime \prime}, 750.1^{\prime \prime}, 936.6^{\prime \prime}$ for the DOP and polarization angle $\Psi$. The lines and colours are as in Figs. 6, 7. The DOP and the magnitude of the polarization angle $\Psi$ remain symmetrical along the source perpendicular to radial direction. 
N. L. S. Jeffrey and E. P. Kontar: Spatially resolved hard X-ray polarization in solar flares
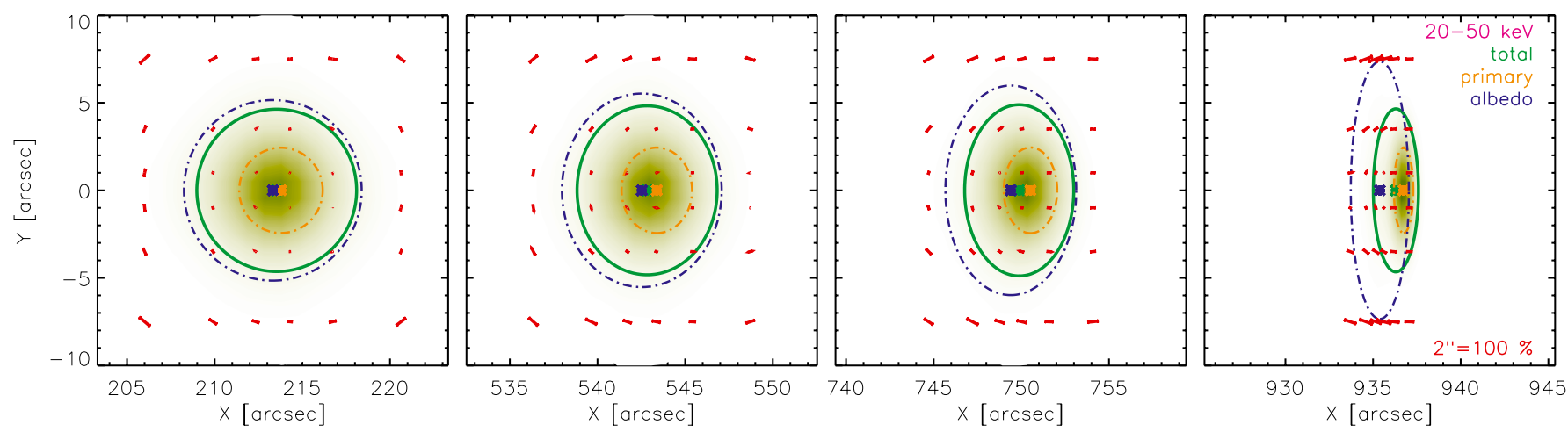

Fig. 9. Total X-ray brightness and polarization maps for the photon distribution created by the $\Delta v=0.5$ electron distribution for a $5^{\prime \prime}$ primary source. The resulting total sources sit at four disk locations of $X=213.1^{\prime \prime}, 542.6^{\prime \prime}, 750.0^{\prime \prime}, 936.5^{\prime \prime}$ at $Y=0^{\prime \prime}$ (corresponding to $\mu \sim 0.97,0.82,0.62,0.22$ ). The green, blue and orange ellipses give the $F W H M$ of the total, albedo and primary sources respectively. Green, blue and orange asterisks give the centroid position of the total, albedo and primary sources. For disk locations closer to the solar centre, the albedo component is the dominant component over the primary component.
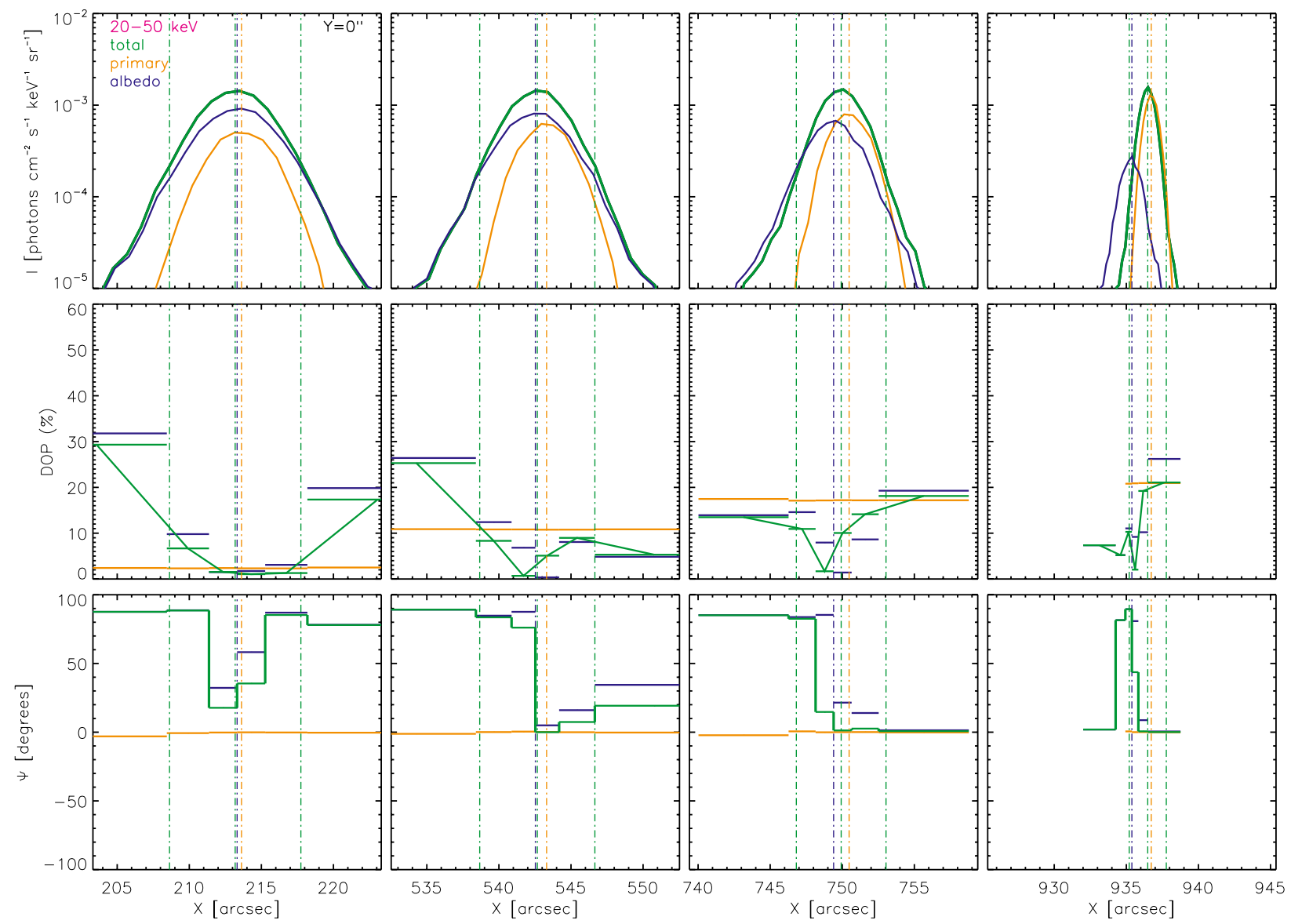

Fig. 10. Radial slices (along $X$ ) through $Y=0^{\prime \prime}$ for the intensity, $I$, the DOP and $\Psi$ for each of the sources in Fig. 9 for the $\Delta v=0.5$ distribution. Again, orange $=$ primary, blue $=$ albedo and green $=$ total. The green dash-dot lines denote the centroid position and the $F W H M$ of the total observed source while the orange and blue lines denote the centroid positions of the primary and albedo sources respectively.

demonstrates that increased beaming towards the photosphere produces smaller, more concentrated and intense albedo patches.

For the $\Delta v=0.5$ distribution, Fig. 10 plots the radial intensity, $I$ (top) (along $X$ at $Y=0^{\prime \prime}$ ). The first two disk locations are albedo dominated, the third disk location has approximately equal contributions from the primary and albedo components and only the disk location closest to the limb is primary dominated. The primary DOP can rise as high as $\sim 20 \%$ at the limb and the primary $\Psi$ is radial at all locations. Figure 13 (top) plots radial intensity slices (along $X$ at $Y=0^{\prime \prime}$ ) for the $\Delta v=0.1$ distribution. As expected, the first three disk locations are albedo dominated and the primary DOP can reach $\sim 40 \%$ at the limb. Again, the primary $\Psi$ is radial at all disk locations.

As with the quasi-isotropic $\Delta v=4.0$ distribution, common trends can be observed across individual sources at particular disk locations and between disk locations for both the $\Delta v=0.5$ and $\Delta v=0.1$ distributions. More importantly for observations and anisotropy deduction purposes, trends between each of the three simulated distributions ( $\Delta v=4.0, \Delta v=0.5$ and $\Delta v=0.1$ ) can be observed, along the radial and perpendicular to radial 

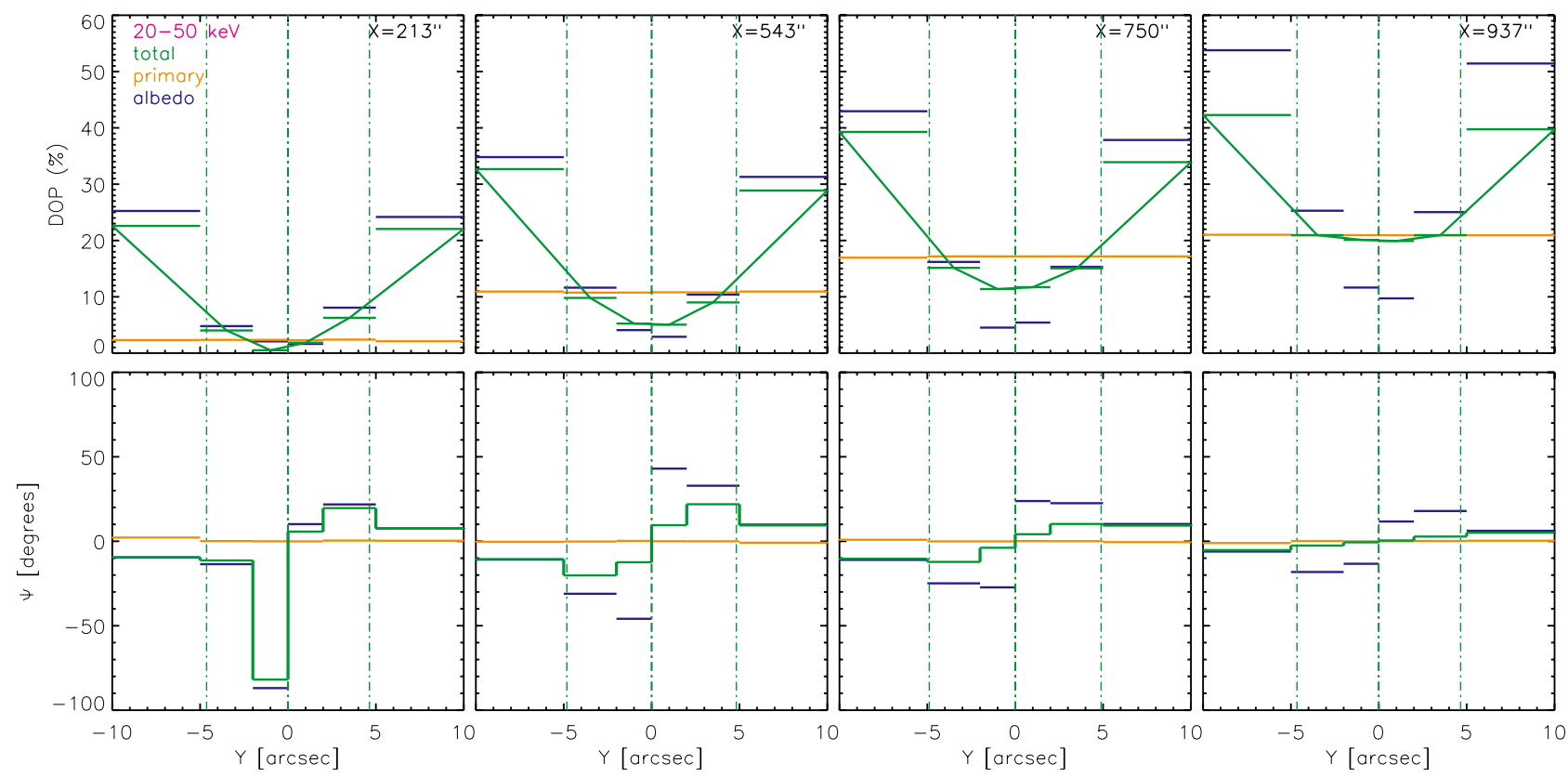

Fig. 11. Perpendicular to radial slices through each of the sources shown in Fig. 9 for the $\Delta v=0.5$ distribution. Each of the perpendicular to radial slices are taken along $Y$ at $X=213.1^{\prime \prime}, 542.6^{\prime \prime}, 750.0^{\prime \prime}, 936.5^{\prime \prime}$ for the DOP and polarization angle $\Psi$. The lines and colours are as in Figs. 9, 10.
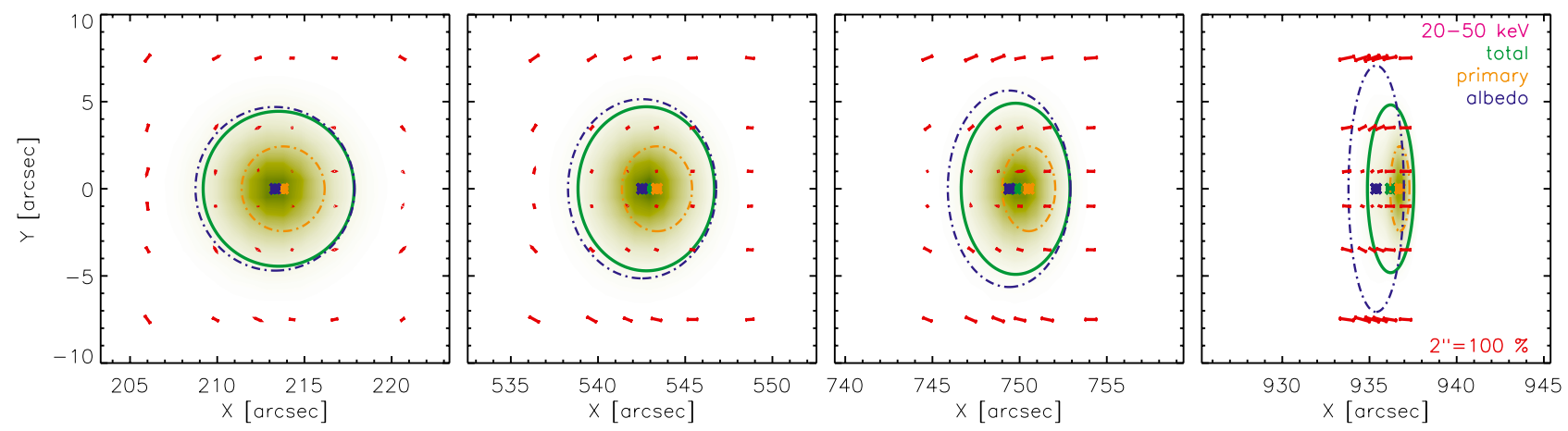

Fig. 12. Total X-ray brightness and polarization maps for the photon distribution created by the $\Delta v=0.1$ electron distribution for a $5^{\prime \prime}$ primary source. The resulting total sources sit at four disk locations of $X=213.0^{\prime \prime}, 542.5^{\prime \prime}, 749.9^{\prime \prime}, 936.4^{\prime \prime}$ at $Y=0^{\prime \prime}$ (corresponding to $\mu \sim 0.97,0.82,0.62,0.22)$. The green, blue and orange ellipses give the $F W H M$ of the total, albedo and primary sources respectively. Green, blue and orange asterisks give the centroid position of the total, albedo and primary sources. At all disk locations the albedo component is the dominant component over the primary component.

directions, at any chosen disk location. The most notable trends are observed in the radial $(X)$ direction, and it is these trends that may help deduce something about the anisotropy of the photon distribution for an HXR source sitting at a given disk location. Trends can be observed at all disk locations, but in this example the patterns are most noticeable in the second and third disk locations plotted. In both of these locations, the disk-centre-side DOP falls with increased beaming while the limb-side DOP rises with increased beaming.

Comparing the third disk location (for example) in Figs. 7, 10 and 13 shows how the radial DOP at the limb-side of the source rises with increased beaming from $\sim 2 \%$ for the $\Delta v=4.0$ distribution to $\sim 18 \%$ for the $\Delta v=0.5$ distribution to $\sim 30 \%$ for the $\Delta v=0.1$ distribution. The radial DOP at the disk-centre-side of the source falls with increased beaming from $\sim 18 \%(\Delta v=4.0)$ to $\sim 14 \%(\Delta v=0.5)$ to $\sim 4 \%(\Delta v=0.1)$. The polarization angle $\Psi$ also produces similar patterns with changing anisotropy. A clear example of this can be observed by comparing the second disk location plotted in Figs. 7, 10 and 13. Along the radial direction, disk-centre-side $\Psi$ generally stays at $\Psi=90^{\circ}$ for all photon anisotropies, while the outer limb-side
$\Psi$ falls significantly with increased beaming, from $\Psi=60^{\circ}$ $(\Delta v=4.0)$ to $\Psi=20^{\circ}(\Delta v=0.5)$ to $\Psi=0^{\circ}(\Delta v=0.1)$.

Therefore, the DOP and $\Psi$ patterns are a clear indication of how spatially resolved polarization measurements could be used to determine the beaming of the photon distribution. It should be noted that a (near) disk-centre source produces a slightly different trend in radial DOP with increasing photon anisotropy. The DOP at the disk-centre-side remains approximately the same for all photon anisotropies while the DOP at the limb-side falls with increased beaming (this is the opposite trend to other disk locations).

Comparing each disk location along the perpendicular to radial direction $(Y)$ in Figs. 8, 11 and 14 shows that greater beaming increases the DOP over the whole extent of the source at any given location (except at the disk centre where the spatially resolved polarization along $Y$ is approximately the same for all three distributions). This spatial increase is most noticeable at limb locations where from the source centre to the source edge, DOP increases from $\sim 0 \%$ to $\sim 20 \%(\Delta v=4.0)$, from $\sim 20 \%$ to $\sim 40 \%(\Delta v=0.5)$ and from $\sim 40 \%$ to $\sim 55 \%(\Delta v=0.1)$. 
N. L. S. Jeffrey and E. P. Kontar: Spatially resolved hard X-ray polarization in solar flares
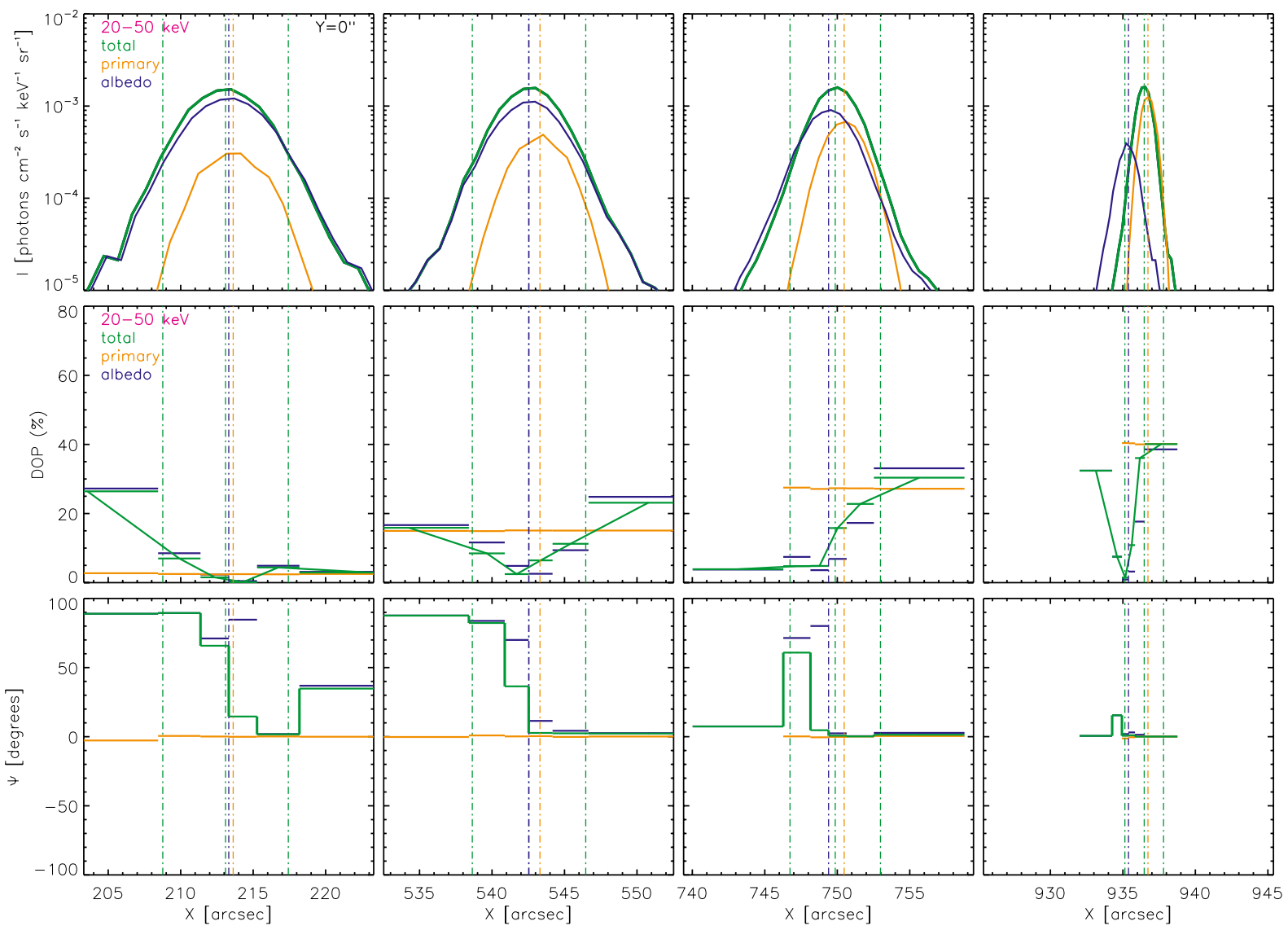

Fig. 13. Radial slices (along $X$ ) through $Y=0^{\prime \prime}$ for the intensity, $I$, the DOP and $\Psi$ for each of the sources in Fig. 12 for the $\Delta v=0.1$ distribution. Again, orange $=$ primary, blue $=$ albedo and green $=$ total. The green dash-dot lines denote the centroid position and the $F W H M$ of the total observed source while the orange and blue lines denote the centroid positions of the primary and albedo sources respectively.
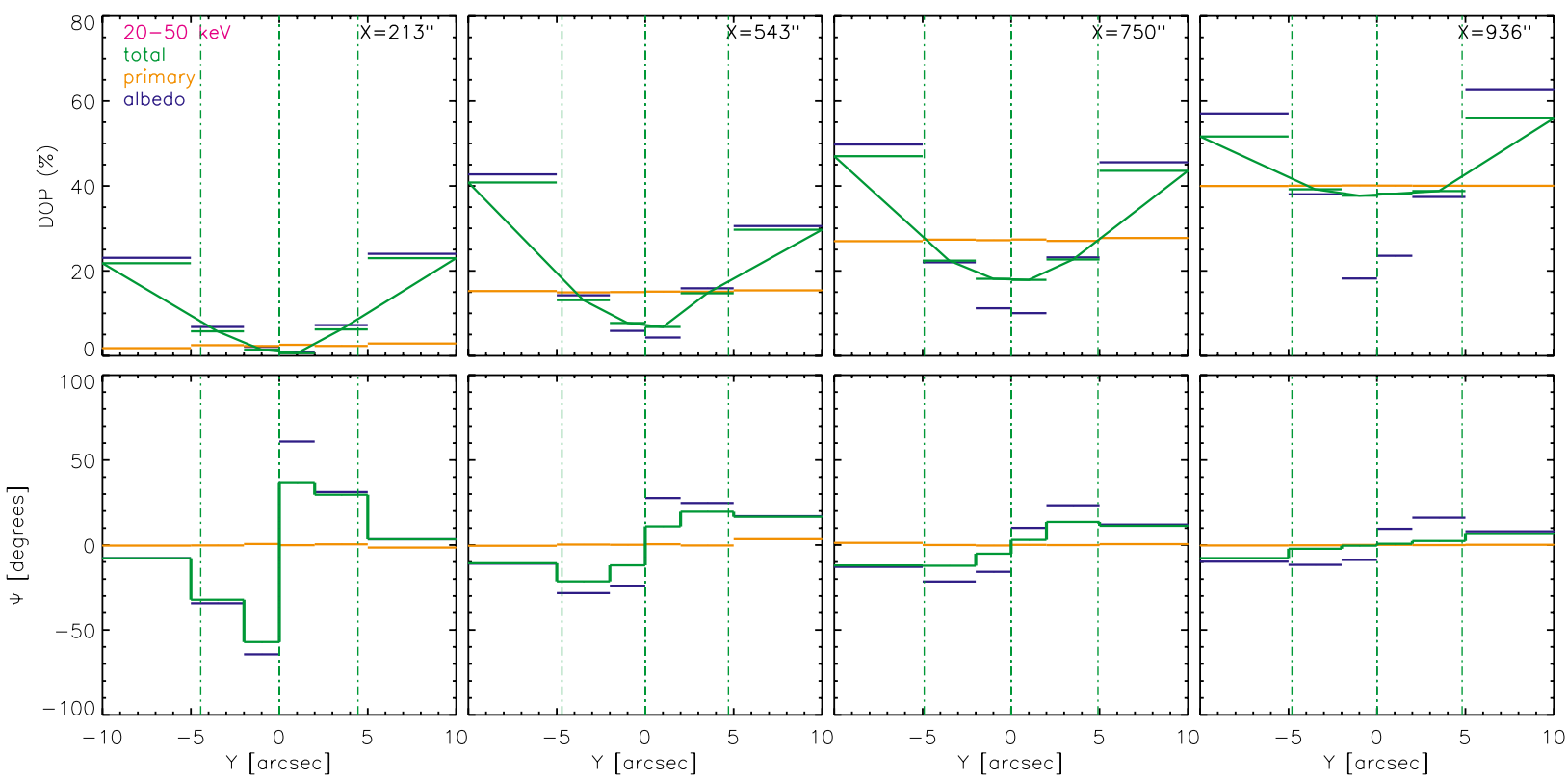

Fig. 14. Perpendicular to radial slices through each of the sources shown in Fig. 12 for the $\Delta v=0.1$ distribution. Each of the perpendicular to radial slices are taken along $Y$ at $X=213.0^{\prime \prime}, 542.5^{\prime \prime}, 749.9^{\prime \prime}, 936.4^{\prime \prime}$ for the DOP and polarization angle $\Psi$. The lines and colours are as in Figs. 12, 13.

\subsection{High energy cutoff in the electron distribution and $H X R$ polarization}

Spatially integrated polarization is dependent on the highest energy in the electron distribution (the high cutoff energy)
(Heristchi 1987). When calculating spatially integrated polarization, Eq. (1) reduces to $D O P=Q$ and Eq. (2) reduces to $\Psi=0.5 \arctan \left(\frac{-0}{-Q}\right)$ as $U$ sums to zero for a single measurement across the entire source. A negative DOP indicates that the polarization angle is parallel to the radial direction $\left(\Psi=0^{\circ}\right)$, while 

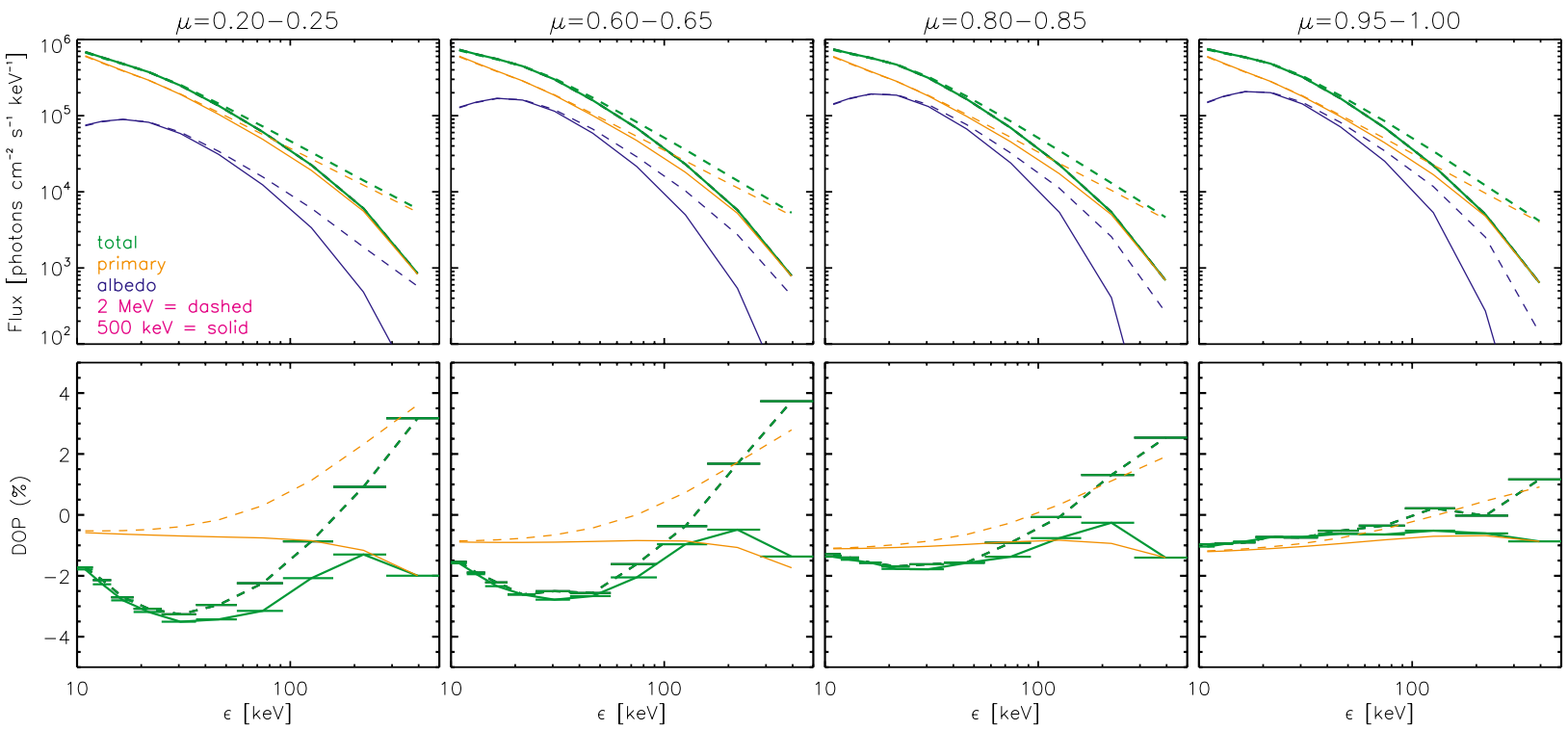

Fig. 15. Changes in flux (top row) and spatially integrated polarization (bottom row) for the total source (green), primary (orange) and albedo (blue) components with photon energy $\epsilon$ for the $\Delta v=4.0$ created photon distribution with $E_{\text {cutoff }}=500 \mathrm{keV}$ (solid lines) and $E_{\text {cutoff }}=2 \mathrm{MeV}$ (dashed lines). Note that the four disk locations shown are the same as those used in Fig. 6. Though there are small changes in the magnitude (DOP) of the polarization, at high photon energies $(\sim 200 \mathrm{keV})$, the polarization direction of the $2 \mathrm{MeV}$ distribution changes from $\Psi=0^{\circ}$ (negative) to $\Psi=90^{\circ}$ (positive), while $\Psi$ for the $500 \mathrm{keV}$ distribution always stays negative.

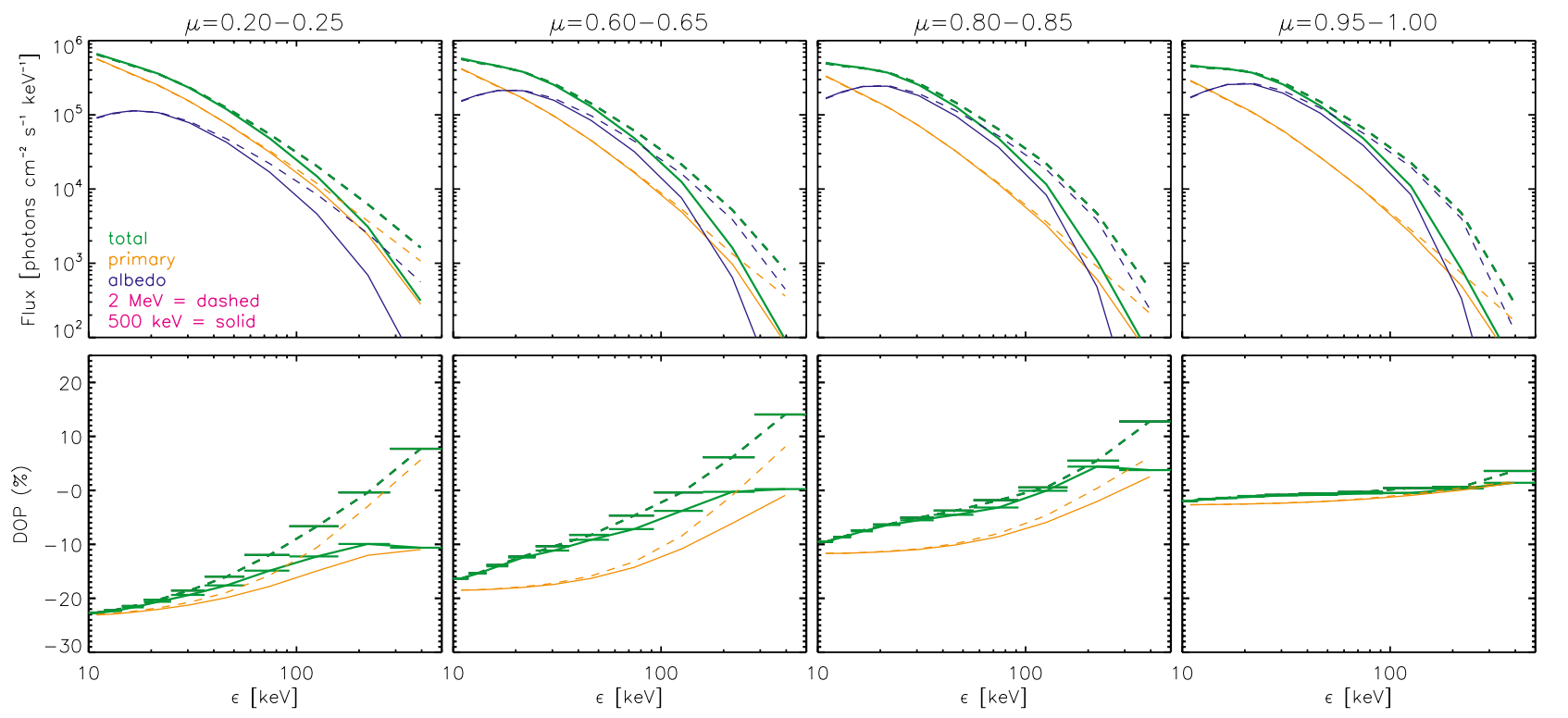

Fig. 16. Changes in flux (top row) and spatially integrated polarization (bottom row) for the total source (green), primary (orange) and albedo (blue) components with photon energy $\epsilon$ for the $\Delta v=0.5$ created photon distribution with $E_{\text {cutoff }}=500 \mathrm{keV}$ (solid lines) and $E_{\text {cutoff }}=2 \mathrm{MeV}$ (dashed lines) for four solar disk locations.

a positive DOP indicates that the polarization angle is perpendicular to the radial direction $\left(\Psi=90^{\circ}\right)$.

For the three electron distributions of $\Delta v=4.0, \Delta v=$ 0.5 and $\Delta v=0.1$, simulations were run with two high cutoff electron energies of $E_{\text {cutoff }}=500 \mathrm{keV}$ and $E_{\text {cutoff }}=$ $2 \mathrm{MeV}$. Figures 15-17 plot the spatially integrated polarization across the total source (green) and the primary source only (orange) against photon energy $\epsilon$ at four disk locations $\mu \in$ [0.20-0.25], [0.60-0.65], [0.80-0.85], [0.95-1.00] for each of the above distributions respectively. The important property to observe here is not the magnitude but the sign of the DOP (or whether $\Psi=0^{\circ}$ or $\Psi=90^{\circ}$ ).
Using an electron distribution with $\Delta v=4.0$ (Fig. 15) and a cutoff energy of $E_{\text {cutoff }}=500 \mathrm{keV}$ produces a photon distribution with a negative DOP at all photon energies and disk locations, while using the same distribution with a cutoff energy of $E_{\text {cutoff }}=2 \mathrm{MeV}$ creates a photon distribution where the DOP changes from negative to positive at $\sim 100-200 \mathrm{keV}$ at all disk locations. During bremsstahlung, in order to conserve energy, electrons with higher energies will scatter through larger angles. When a photon is scattered through a large angle, its polarization is more likely to be directed perpendicular to the plane of emission $\left(\Psi=90^{\circ}\right)$ rather than parallel to the plane of emission $\left(\Psi=0^{\circ}\right)$. Therefore, a change in the direction of polarization 
N. L. S. Jeffrey and E. P. Kontar: Spatially resolved hard X-ray polarization in solar flares
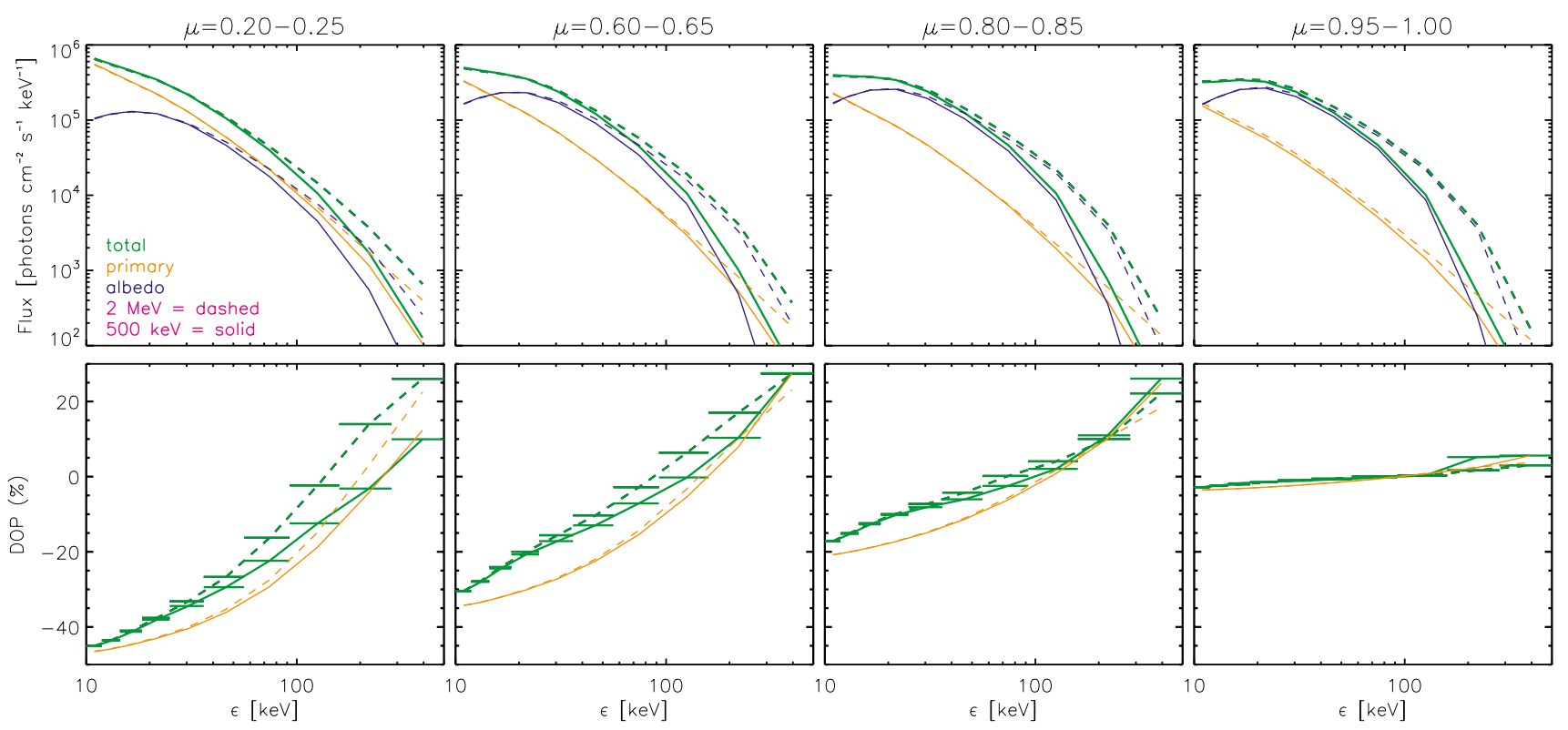

Fig. 17. Changes in flux (top row) and spatially integrated polarization (bottom row) for the total source (green), primary (orange) and albedo (blue) components with photon energy $\epsilon$ for the $\Delta v=0.1$ created photon distribution with $E_{\text {cutoff }}=500 \mathrm{keV}$ (solid lines) and $E_{\text {cutoff }}=2 \mathrm{MeV}$ (dashed lines) for four solar disk locations.

(from $\Psi=0^{\circ}$ to $\Psi=90^{\circ}$ ) indicates the presence of higher energies in the electron distribution, greater than $\sim 1 \mathrm{MeV}$.

As the beaming of the electron distribution increases (the $\Delta v=0.5$ and $\Delta v=0.1$ distributions), we see that the above statement does not hold and it becomes more likely that both electron distributions with cutoff energies of either $E_{\text {cutoff }}=500 \mathrm{keV}$ and $E_{\text {cutoff }}=2 \mathrm{MeV}$ will produce photons with $\Psi=90^{\circ}$. For the $\Delta v=0.5$ distribution (Fig. 16), we see that as the source moves towards the solar centre, the photon distribution created by the $E_{\text {cutoff }}=500 \mathrm{keV}$ also produces photons at higher energies (again $\sim 100-200 \mathrm{keV}$ ) with $\Psi=90^{\circ}$. For the very beamed $\Delta v=0.1$ distribution (Fig. 17), both the $E_{\text {cutoff }}=500 \mathrm{keV}$ and $E_{\text {cutoff }}=2 \mathrm{MeV}$ distributions produce high $(\sim 100-200 \mathrm{keV})$ energy photons with $\Psi=90^{\circ}$ at all disk locations.

Therefore using the direction of $\Psi$ as an indicator for high energies in the electron distribution becomes less and less useful as the beaming of the photon (electron) distribution increases. The increased beaming causes lower and lower energy photons to scatter at larger angles, especially at locations closer to the solar centre, hence producing photons with $\Psi=90^{\circ}$ in the $E_{\text {cutoff }}=500 \mathrm{keV}$ distributions. Note that this method is only useful when the beaming of the photon distribution has already been determined.

\section{Discussion and conclusions}

The simulation results show that Compton backscattering produces a clear and distinct albedo polarization pattern across an HXR source at energies of 20-50 keV. Trends can be observed for both of the measured polarization parameters, DOP and $\Psi$, and are clear indications of the existence of an albedo component in comparison with the constant, radial polarization of the primary emission. This means that spatially resolved polarization can be used to probe structure within HXR footpoint sources, helping us distinguish between the bremsstrahlung source and the albedo source. More importantly, at a single disk location, spatially resolved DOP and $\Psi$ exhibit clear variations with changing photon directivity, along both the radial and perpendicular to radial directions and can be used to determine the anisotropy of the electron distribution. Therefore, to take advantage of these properties requires reliable polarization measurements with an angular resolution of $\sim 5^{\prime \prime}-10^{\prime \prime}$ over the peak albedo energies of $\sim 20-50 \mathrm{keV}$.

The simulations also suggest that for $\sim$ isotropic HXR sources, spatially integrated polarization angle measurements, $\Psi$, from low to high energies, with consideration of the changes due to albedo, could help indirectly infer the highest energy in the electron distribution. For near isotropic sources implied by RHESSI X-ray observations (Kašparová et al. 2007; Kontar \& Brown 2006), changes in $\Psi$ from the radial $\left(\Psi=0^{\circ}\right)$ to the perpendicular to radial direction $\left(\Psi=90^{\circ}\right)$ may help indicate the presence of high energy electrons $(\sim \geq 1 \mathrm{MeV})$ present in the electron distribution, if the photon anisotropy is known. Changes in spatially integrated DOP measurements, from low to high energies, will also help determine the anisotropy of the photon distribution.

Currently, when observing solar flares we do not have the instrumentation required to produce spatially resolved polarization measurements. It is doubtful whether near future missions such as the Gamma-Ray Imager/Polarimeter for Solar Flares (GRIPS) (Shih et al. 2009) may have the capability to measure polarization over albedo energies, even though it should be able to measure polarization across $12.5^{\prime \prime}$. The best polarization measurements are likely to be over the range of $150-650 \mathrm{keV}$ with a $\sim 4 \%$ minimum detectable polarization (Shih et al. 2009). At energies greater than $\sim 100 \mathrm{keV}$, the albedo flux drops off steeply, so it is very unlikely that any albedo component could be detected at these energies and the polarization across the observed HXR source would only be from the bremsstrahlung emission. Therefore, good polarization measurements at 150$650 \mathrm{keV}$ from flares with high fluxes will give us a direct measurement of the primary component and DOP $/ \Psi$ measurements at these energies may be used to infer the high energy cutoff in the electron distribution, for relatively isotropic distributions.

Acknowledgements. N.L.S.J. and E.P.K. are grateful to A. Gordon Emslie for the use of his bremsstrahlung cross-section code and Hugh Hudson, Albert 
Shih and Gordon Holman for useful comments and discussions. N.L.S.J. is funded by STFC and SUPA scholarships. E.P.K. gratefully acknowledges the financial support by the Leverhulme Trust (EPK), STFC Rolling Grant, and by the European Commission through the HESPE (FP7-SPACE-2010-263086) Network acknowledged.

\section{Appendix A: Compton scattering and new scattering angles and energies}

In the Monte Carlo simulations, when a Compton scattering occurs, the properties of the outgoing photon: energy $\epsilon$, polar scattering angle $\theta_{\mathrm{S}}$, azimuthal scattering angle $\phi_{\mathrm{S}}$ and the two linear polarisation parameters $Q$ and $U$ need to be updated. New polar scattering angles $\theta_{\mathrm{S}}$ for each photon can be found by integrating the Klein-Nishina differential cross section over $\phi_{\mathrm{S}}$ to produce a differential cross-section that is only dependent on $\epsilon$ and $\theta_{\mathrm{S}}$. This is the Klein-Nishina (Klein \& Nishina 1929) differential crosssection for a completely unpolarised isotropic beam and is given by,

$\frac{\mathrm{d} \sigma_{\mathrm{c}}\left(\theta_{\mathrm{S}}, \epsilon\right)}{\mathrm{d} \Omega}=\frac{1}{2} r_{0}^{2}\left(\left(\frac{\epsilon}{\epsilon_{0}}\right)^{3}+\frac{\epsilon_{0}}{\epsilon}-\left(\frac{\epsilon}{\epsilon_{0}}\right)^{2} \sin ^{2} \theta_{\mathrm{S}}\right)$.

Since MC simulations operate by drawing numbers randomly from a given distribution, this means that $\theta_{\mathrm{S}}$ can be easily found by matching each value of $\theta_{\mathrm{S}} \in\left[0^{\circ}, 180^{\circ}\right]$ with a number $\zeta_{\theta} \in[0,1]$ for every value of $\epsilon$ using,

$\zeta_{\theta}=\frac{2 \pi}{\sigma_{\mathrm{c}}} \int_{0}^{\theta_{\mathrm{S}}} \frac{\mathrm{d} \sigma_{\mathrm{c}}\left(\theta_{\mathrm{S}}, \epsilon\right)}{\mathrm{d} \Omega} \mathrm{d} \theta_{\mathrm{S}}$

where $\sigma_{\mathrm{c}}$ is the total Compton scattering cross-section. New values of $\theta_{\mathrm{S}}$ are simply drawn at each scattering using the photon energy before scattering and a random, uniform number between 0 and 1 .

Once the new scattering angles $\theta_{\mathrm{S}}$ are obtained then the new photon energy $\epsilon$ can be easily found using,

$\epsilon=\frac{\epsilon_{0}}{1+\frac{\epsilon_{0}}{m c^{2}}\left(1-\cos \theta_{\mathrm{S}}\right)}$.

\section{Appendix B: Compton scattering and photon polarization states}

If the photon distribution is completely isotropic and unpolarised then the azimuthal scattering angle $\phi_{\mathrm{S}}$ can just be sampled from a uniform distribution between 0 and $2 \pi$, but this is not true for the more general polarization dependent case. The probability density function of obtaining a value of $\phi_{\mathrm{S}}$ between $\phi_{\mathrm{S}}$ and $\phi_{\mathrm{S}}+$ $\mathrm{d} \phi_{\mathrm{S}}$ can be described by:

$$
\begin{aligned}
P\left(\phi_{\mathrm{S}}\right) & =\frac{1}{2 \pi} \frac{\mathrm{d} \sigma_{\mathrm{c}}\left(\epsilon, \theta_{\mathrm{S}}, \phi_{\mathrm{S}}\right) / \mathrm{d} \Omega}{\mathrm{d} \sigma_{\mathrm{c}}\left(\epsilon, \theta_{\mathrm{S}}\right) / \mathrm{d} \Omega} \\
& =\frac{1}{2 \pi} \frac{\frac{\epsilon_{0}}{\epsilon}+\frac{\epsilon}{\epsilon_{0}}-\sin ^{2} \theta_{\mathrm{S}}\left(1-Q \cos 2 \phi_{\mathrm{S}}-U \sin 2 \phi_{\mathrm{S}}\right)}{\frac{\epsilon_{0}}{\epsilon}+\frac{\epsilon}{\epsilon_{0}}-\sin ^{2} \theta_{\mathrm{S}}},
\end{aligned}
$$

with the maximum value of this function given by:

$P_{\max }\left(\phi_{\mathrm{S}}\right)=\frac{1}{2 \pi} \frac{\frac{\epsilon_{0}}{\epsilon}+\frac{\epsilon}{\epsilon_{0}}-\sin ^{2} \theta_{\mathrm{S}}\left(1-\sqrt{Q^{2}+U^{2}}\right)}{\left(\frac{\epsilon_{0}}{\epsilon}+\frac{\epsilon}{\epsilon_{0}}-\sin ^{2} \theta_{\mathrm{S}}\right)}$.

Firstly, a value of $\phi_{\mathrm{S}}$ is sampled between 0 and $2 \pi$. The condition that $P\left(\phi_{\mathrm{S}}\right)<P_{\max }\left(\phi_{\mathrm{S}}\right)$ is then used to accept a value of $\phi_{\mathrm{S}}$ and provides a method for sampling values of $\phi_{\mathrm{S}}$ for each photon,

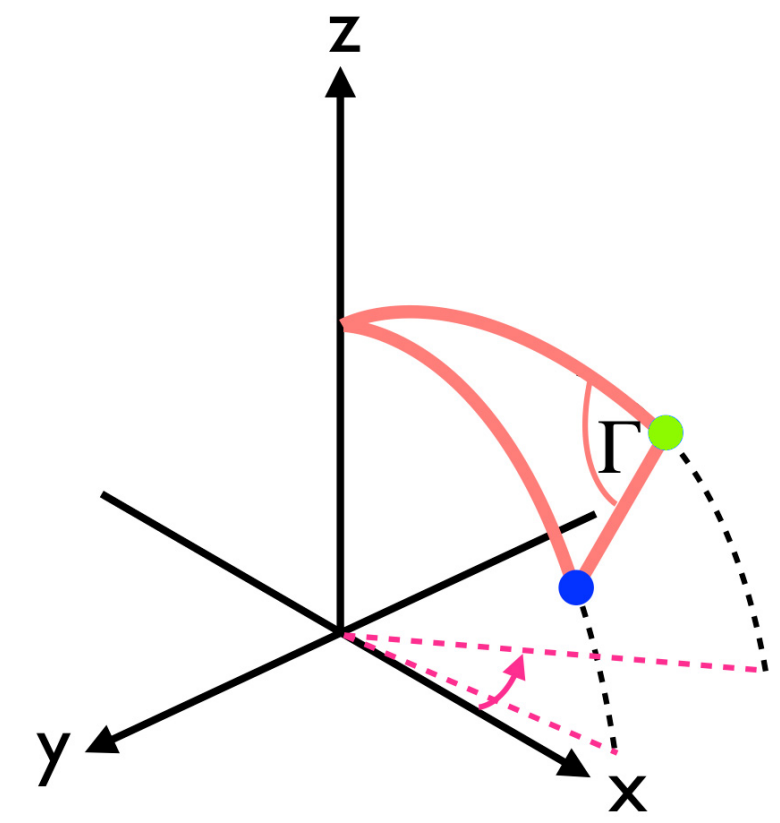

Fig. B.1. The position of the photon before scattering (blue) and after scattering (green) and the angle $\Gamma$ that determines the final rotation of the Stokes parameters back into the frame of the source from the scattering frame.

using the new values $\theta_{S}$ and $\epsilon$ already calculated for each photon/photon packet. This method is repeated until the condition is satisfied for each photon and each photon is provided with an azimuthal scattering angle (Salvat et al. 2008).

Due to Compton scattering, the Stokes parameters have to be updated using the scattering matrix $T$ (McMaster 1961; Bai \& Ramaty 1978)

$T\left(\epsilon, \theta_{\mathrm{S}}\right)=\left(\begin{array}{ccc}\frac{\epsilon}{\epsilon_{0}}+\frac{\epsilon_{0}}{\epsilon}+\sin ^{2} \theta_{\mathrm{S}} & \sin ^{2} \theta_{\mathrm{S}} & 0 \\ \sin ^{2} \theta_{\mathrm{S}} & \cos ^{2} \theta_{\mathrm{S}}+1 & 0 \\ 0 & 0 & 2 \cos \theta_{\mathrm{s}}\end{array}\right)$.

Before a scattering, the Stokes parameters have to be rotated by $\phi_{\mathrm{S}}$ so that they are defined relative to the plane of scattering. The Stokes parameters before scattering are rotated by the rotation matrix $M\left(\phi_{\mathrm{S}}\right)$ given by:

$M\left(\phi_{\mathrm{S}}\right)=\left(\begin{array}{ccc}1 & 0 & 0 \\ 0 & \cos 2 \phi_{\mathrm{S}} & \sin 2 \phi_{\mathrm{S}} \\ 0 & -\sin 2 \phi_{\mathrm{S}} & \cos 2 \phi_{\mathrm{S}}\end{array}\right)$

After a scattering, the Stokes parameters have to be rotated again so that they are defined relative to the starting position of the source. The Stokes parameters after scattering are rotated by the rotation matrix $M(-\Gamma)$.

$M(-\Gamma)=\left(\begin{array}{ccc}1 & 0 & 0 \\ 0 & \cos -2 \Gamma & \sin -2 \Gamma \\ 0 & -\sin -2 \Gamma & \cos -2 \Gamma\end{array}\right)$,

where

$\cos \Gamma= \pm \frac{w^{\prime}-\cos \theta_{\mathrm{s}} w}{\sqrt{1-\cos ^{2} \theta_{\mathrm{s}}} \sqrt{1-w^{2}}}$,

$w$ and $w^{\prime}$ are the current and previous $z$ direction cosines respectively (Hovenier \& van der Mee 1983). The \pm in Eq. (B.6) is 
present due to the negative sign being used when $\pi \leq \phi_{s} \leq 2 \pi$ and the positive sign for $0 \leq \phi_{\mathrm{S}}<\pi$. The angle $\Gamma$ is shown in Fig. B.1. $\Gamma$ is the angle between the scattering plane and the normal plane in the frame of the source. Therefore during a Compton scattering the order of the rotations on the Stokes vector $[I Q U]$ is $M\left(\phi_{\mathrm{S}}\right) T\left(\epsilon, \theta_{\mathrm{S}}\right) M(-\Gamma)$.

\section{References}

Asplund, M., Grevesse, N., Sauval, A. J., \& Scott, P. 2009, ARA\&A, 47, 481 Bai, T., \& Ramaty, R. 1978, ApJ, 219, 705

Balucinska-Church, M., \& McCammon, D. 1992, ApJ, 400, 699

Battaglia, M., \& Kontar, E. P. 2011, ApJ, 735, 42

Bloser, P. F., Legere, J. S., McConnell, M. L., et al. 2009, Nucl. Inst. Meth. Phys. Res. A, 600, 424

Boggs, S. E., Coburn, W., \& Kalemci, E. 2006, ApJ, 638, 1129

Brown, J. C. 1972, Sol. Phys., 26, 441

Chandrasekhar, S. 1960, Radiative transfer (Dover: New York)

Elwert, G., \& Haug, E. 1970, Sol. Phys., 15, 234

Emslie, A. G., Bradsher, H. L., \& McConnell, M. L. 2008, ApJ, 674, 570

Gluckstern, R. L., \& Hull, M. H. 1953, Phys. Rev., 90, 1030

Haug, E. 1972, Sol. Phys., 25, 425

Henoux, J. C. 1975, Sol. Phys., 42, 219

Heristchi, D. 1987, ApJ, 323, 391

Holman, G. D., Aschwanden, M. J., Aurass, H., et al. 2011, Space Sci. Rev., 159, 107

Hovenier, J. W., \& van der Mee, C. V. M. 1983, A\&A, 128, 1

Kašparová, J., Kontar, E. P., \& Brown, J. C. 2007, A\&A, 466, 705

Klein, O., \& Nishina, T. 1929, Zeitschrift fur Physik, 52, 853

Kontar, E. P., \& Brown, J. C. 2006, ApJ, 653, L149

Kontar, E. P., \& Jeffrey, N. L. S. 2010, A\&A, 513, L2

Kontar, E. P., MacKinnon, A. L., Schwartz, R. A., \& Brown, J. C. 2006, A\&A, 446, 1157

Kontar, E. P., Hannah, I. G., \& MacKinnon, A. L. 2008, A\&A, 489, L57
Kontar, E. P., Hannah, I. G., Jeffrey, N. L. S., \& Battaglia, M. 2010, ApJ, 717, 250

Kontar, E. P., Brown, J. C., Emslie, A. G., et al. 2011, Space Sci. Rev., 159, 301 Langer, S. H., \& Petrosian, V. 1977, ApJ, 215, 666

Leach, J., \& Petrosian, V. 1983, ApJ, 269, 715

Lemen, J. R., Chanan, G. A., Hughes, J. P., et al. 1982, Sol. Phys., 80, 333

Massone, A. M., Emslie, A. G., Kontar, E. P., et al. 2004, ApJ, 613, 1233

McConnell, M. L., Smith, D. M., Emslie, A. G., et al. 2004, Adv. Space Res., 34,462

McConnell, M. L., Ryan, J. M., Smith, D. M., et al. 2007, in BAAS, 38, AAS Meet. Abst., \#210, 211

McMaster, W. H. 1961, Rev. Mod. Phys., 33, 8

Morrison, R., \& McCammon, D. 1983, ApJ, 270, 119

Mrozek, T., \& Kowalczuk, J. 2010, Central Eur. Astrophy. Bull., 34, 57

Muleri, F., Bellazzini, R., Brez, A., et al. 2009, J. Inst., 4, 11002

Nakada, M. P., Neupert, W. M., \& Thomas, R. J. 1974, Sol. Phys., 37, 429

Poutanen, J., Nagendra, K. N., \& Svensson, R. 1996, MNRAS, 283, 892

Prato, M., Emslie, A. G., Kontar, E. P., Massone, A. M., \& Piana, M. 2009, ApJ, 706, 917

Saint-Hilaire, P., Krucker, S., \& Lin, R. P. 2010, ApJ, 721, 1933

Salvat, F., Fernandez-Varea, J. M., \& Sempau, J. 2008, France: OECD Nuclear Energy Agency, Issy-les-Moulineaux

Santangelo, N., Horstman, H., \& Horstman-Moretti, E. 1973, Sol. Phys., 29, 143

Shih, A. Y., Lin, R. P., Hurford, G. J., et al. 2009, in AAS/Solar Physics Division Meeting, 40, 18.10

Stokes, G. G. 1852, Trans. Cambridge Phil. Soc., 9, 399

Suarez-Garcia, E., Hajdas, W., Wigger, C., et al. 2006, Sol. Phys., 239, 149

Tindo, I. P., Ivanov, V. D., Mandel'Stam, S. L., \& Shuryghin, A. I. 1970, Sol. Phys., 14, 204

Tindo, I. P., Ivanov, V. D., Valníček, B., \& Livshits, M. A. 1972, Sol. Phys., 27, 426

Tindo, I. P., Shurygin, A. I., \& Steffen, W. 1976, Sol. Phys., 46, 219

Tomblin, F. F. 1972, ApJ, 171, 377

Vernazza, J. E., Avrett, E. H., \& Loeser, R. 1981, ApJS, 45, 635

Zharkova, V. V., Brown, J. C., \& Syniavskii, D. V. 1995, A\&A, 304, 284

Zharkova, V. V., Kuznetsov, A. A., \& Siversky, T. V. 2010, A\&A, 512, A8 